\title{
Les identifications de Gayōmart à l'époque islamique
}

\author{
Par SVEN S. HARTMAN
}

A l'arrivée de l'islam en Iran, Gayōmart y était le premier homme'. Les musulmans avaient cependant un autre premier homme, à savoir Adam. Ainsi un problème se présenta immédiatement et pour les musulmans et pour les non-musulmans : Gayōmart, était-il le premier homme, ou est-ce Adam qui a eu ce "privilège ", ou bien Gayōmart et Adam sont-ils le même personnage? Il semble peut-être que la solution la plus proche serait à identifier Gayōmart et Adam, ce que l'on a parfois aussi fait. Mais cette solution n'explique pourtant qu'une partie restreinte du problème et comporte elle-même la naissance de beaucoup de nouveaux au sujet de la géographie religieuse, la chronologie, la généalogie et l'histoire primitive en général. Car et Gayōmart et l'Adam islamique ont été unis organiquement à leur propre religion. La situation en ce qui concerne Gayōmart a aussi impliqué une précision de la position en faveur ou contre la religion préislamique de l'Iran. Et prendre position au sujet d'Adam a dépendu de l'attitude d'une personne vis-à-vis de l'islam. La question n'a pas été exclusivement religieuse mais aussi politique, car les deux figures ont aussi représenté deux peuples, à savoir les Persans et les Arabes.

Après cela on ne s'étonnera pas qu'au temps islamique Gayōmart se soit identifié avec beaucoup de figures par différents groupes ou personnes.

On ne peut pas dire que les identifications de Gayōmart ont été examinées scientifiquement. Christensen en a traité un tiers sur une page de son livre «Les types du premier homme et du premier roi $»^{2}$. Mais il semble les regarder comme d'inventions arbitraires. Il dit à peu près ainsi : L'idée de Gayōmart premier homme ne disparaît pas, mais comme les moslims ont déjà leur Adam, les auteurs islamiques cherchent à tirer au clair la relation entre ces deux types du premier homme. La supposition qui se présente d'abord à l'esprit est celle que Gayōmart était un autre nom d'Adam .... 
Et puis Christensen fait mention des identifications différentes qu'il a trouvées; mais il n'observe pas la tension qu'il y a entre certaines identifications, une tension qui reflète d'intérêts différents en ce qui concerne la religion et la politique. Déjà d'Herbelot ${ }^{3}$ a cependant découvert le fait que les identifications ne sont pas des créations arbitraires. Car il fait la distinction entre les identifications antédiluviennes créées par les Persans et celles postdiluviennes créées par les Arabes. Cette distinction n'est pas tout à fait correcte, mais elle indique un fait important, à savoir la différence entre les identifications des Persans et celles des Arabes. (En passant nous avons nous-mêmes traité ces identifications de Gayōmart dans notre thèse Gayōmart. Etude sur le syncrétisme dans l'ancien Iran.)

Dans le présent article nous allons examiner chacune des identifications de Gayōmart à l'époque islamique en cherchant à déterminer a) son auteur, b) sa date d'origine, c) sa cause, c.-à-d. le point de contact entre Gayōmart et la figure avec laquelle il a été identifié. Puis dans chaque cas nous allons démontrer quelles sont les suites de l'identification en ce qui concerne l'échange de qualités entre Gayōmart et celui avec qui il a été identifié.

I. Gayōmart comme un ğinn. Cette conception est indiquée dans un livre d'Ibn Habīb4 (mort en 859 ) qui cite (Hišām) ibn al-Kalbī (mort en 820). Elle revient plusieurs siècles plus tard dans le livre Bahğat al-tawārīh (des années 1456-1457) de Sukr Allāh ${ }^{5}$. Celui-ci dit que cette identification émane "des savants du peuple de l'islam »6. Il s'agit ainsi de mahométans et Arabes.

Le contexte de cette identification chez Ibn Habỉb révèle une polémique contre les Persans : On dit que Hišām ibn al-Kalbī a divisé les rois du monde dans deux groupes principaux : l'un étant des ğinn et l'autre des hommes. Dans le premier groupe nous trouvons dans cet ordre : Gayōmart (جيوسرت), Taḩmōruf et Hošang (أوشينك). Le dernier groupe commence par Ğamšēd (جماد), que l'on compte parmi les enfants de Caïn-Qābīl

Si l'on regarde Gayōmart comme un ğinn, ce n'est pas une conception persane. Dans la littérature pehlevie on le représente souvent comme un homme et même comme le premier homme9. Si Hišām ibn al-Kalbī malgré cela traite Gayōmart comme un ğinn, ceci doit être une expression du fait que Hišãm a été un mahométan arabe. Pour résoudre les désaccords entre 
les conceptions arabes et persanes il déclare indirectement les rapports persans mensongers: Gayōmart n'a pas été un homme, ni le premier homme non plus. - Mais pourquoi a-t-on fait de lui un ǧinn? - Les textes ne le disent pas, mais on a probablement voulu polémiser contre les mages qui ont prétendu que Gayōmart a été le premier homme et qu'Adam n'a été qu'un descendant de Gayōmart. Cette supposition est soutenue par le fait que Hišām ibn al-Kalbī après avoir fait mention des trois premiers rois persans dit que les mages racontent d'Adam, que celui-ci est fils de Hōšang ${ }^{10}$. - Les mahométans ont alors pu répondre à ces mages : "Si les dits trois rois persans ont vraiement vécu avant Adam - comme vous prétendez ils ont été des ǧinn, car avant Adam il n'y avait pas d'hommes mais seulement des ǧinn. " Et pour cela on a pu se référer au Coran XV, $27 \mathrm{~s}$.

Comme exemple des suites de cette identification nous pouvons lire ce que d'Herbelot cite de "L'Histoire de Tahmurath en Turc " : dans l'épitaphe de Kaiumarath, premier Roi de Perse, \& Empereur de tout l'Orient, il est fait mention de Gián-Ben-Gián en cette manière : "Qu'est devenu le peuple de Gián, fils de Gián? Regarde ce que le temps en a fait. „11

2. Gayōmart comme fils ou descendant de l'Adam, « qui avait été créé plus tôt qu'Adam, le choisi (d'Allāh) »12. S̆ulrr Allāh, notre seule source de cette identification, ne nous dit pas quels sont ses auteurs, mais il prend lui-même position contre elle en disant : "Cette opinion est une pure erreur parce que l'intervalle entre cet Adam-là et Adam, le choisi (d'Allāh), est de cent cinquante mille ans. Il n'est jamais admis par la raison que l'homme ait cette (grande) longévité alors que (toutes) les vies de ces "Adam » avec leur postérité n'étaient plus que de dix mille (ans). »13

Peut-être cette identification a son origine parmi ceux qui s'appellent Indiens d'après Šahrastānīi ${ }^{14}$ et Murtaḍāi ${ }^{15}$ et qui ont eu des traditions sur Gayōmart. Car les grands chiffres, et l'allusion à plus d'un genre humain pourraient convenir aux spéculations indiennes sur les yuga et les kalpa.

3. Gayōmart comme Aloros ${ }^{16}$. Selon Bérose ${ }^{17}$ Aloros était le premier roi antédiluvien des Chaldéens. Nous savons maintenant que son nom sumérien était Alulim ${ }^{18}$. La forme persane du nom, اللوروس،, est évidemment venue du grec "A $\lambda \omega p o s$ et probablement par l'intermédiaire des chrétiens syriaques parce que ceux-ci (de la même façon que notre source persane) ont eux-mêmes 
raconté d'Aloros qu'il a été roi des enfants de Seth et ennemi des enfants de Caïn, et parce que les syriaques ont d'ailleurs très souvent procuré de la littérature grecque aux Persans ${ }^{19}$.

Ğūzŏganin raconte que les enfants de Seth choisirent les rois chaldéens de sorte que ceux-ci les délivrassent de l'oppression des enfants de Caïn. Le premier de ces rois " portait le nom d'Aloros en grec, et il (c.-à-d. le nom) signifie celui que les non-Arabes et les Persans nomment Gayōmart et dont le surnom était Gilšāh. Ce fut donc le premier des rois des Chaldéens. Et l'on nomme aussi cette dynastie des rois les Pīš-dād'iens. $»^{20}$

Les auteurs de cette identification sont alors les non-Arabes et les Persans.

Notre source persane date de 1260 après J.-C. ${ }^{21}$ Mais l'identification en question doit être plus ancienne. Car il y a des indications, indirectes, c'est vrai, mais très évidentes, qu'un autre roi pīšdād'ien, à savoir Tahymōruf, a été identifié avec Sisouthros ${ }^{22}$, le dernier des rois antédiluviens chaldéens. Déjà Windischmann ${ }^{23}$ a souligné la ressemblance entre le récit de Hamzah sur Tahmōruf et celui de Bérose sur Sisouthros, et il dit avec raison : «Die Übereinstimmung beider Erzählungen von Tahmuraf und von Xisuthros ist so augenfällig, dass sie keines Beweises bedarf ... $\prime^{24}$ Par là nous pouvons dater l'identification de pīšdād'iens avec des rois chaldéens : Le récit de Hamzah sur Tahmōruf qui - comme Sisouthros — cacha des livres pour les garder pendant le déluge, a pour source l'astronome 'Abū $\mathrm{Ma}^{`} \mathrm{Šar}^{25}$, qui mourut en $886^{26}$. Cette sorte d'identifications doit par conséquent dater avant cette année.

La cause de cette identification dépend naturellement du fait qu'on a parfois regardé les Chaldéens comme Persans ${ }^{27}$. La conséquence en est alors : le premier Chaldéen $=$ le premier Persan, c.-à-d. Aloros $=$ Gayōmart.

Comme suite de cette identification Gayōmart a pu être roi des enfants de Seth et ennemi des enfants de Caïn, exactement comme c'est le cas avec l'Aloros dont parlent les chrétiens syriaques.

Chez Bérose, Aloros n'avait rien à faire avec les enfants de Seth ni ceux de Cain, ou avec l'histoire biblique en général. Mais l'Aloros avec lequel Gayōmart a été identifié a appartenu à cette histoire et a alors au moins fait partie d'un syncrétisme antérieur. C'est pourquoi même Gayōmart, identifié avec cet Aloros, est mis en relation avec les personnes de l'histoire biblique, et devenu lui-même un descendant d'Adam. C'est pourquoi aussi 
cette identification semble parfois équivaloir à certaines ayant rapport à un fils ou à un descendant d'Adam sans que l'on nomme le nom d'Aloros ${ }^{28}$.

4. Gayōmart existant deux ou plusieurs générations avant Adam. Nous n'avons qu'une preuve indirecte de l'idée que Gayōmart a vécu deux ou plusieurs générations avant Adam. C'est Ibn Habïb (mort en 859) qui citant Hišām ibn al-Kalbì dit que «certains des mages disent qu'il (c.-à-d. Adam) est Adam, fils de 'Ušyank $»^{29}$.

Gayōmart et Hōšang ont peut-être à l'origine fait partie de religions différentes ${ }^{30}$. Mais dans la littérature pehlevie et d'ailleurs dans toute la littérature ultérieure ils sont proches parents, et presque toujours c'est Gayōmart qui est alors ancêtre de Hōšangi. Si chez Ibn Habỉb Hōšang est le père d'Adam, Gayōmart est probablement reconnu comme existant au moins deux générations avant Adam.

Comme fils de Hōšang, Adam a sans doute perdu sa qualité de premier homme. La conception d'un tel Adam a naturellement son origine chez les mages de la Perse à une époque où ceux-ci ont toujours eu la force nécessaire pour polémiser contre l'islam et déclarer les mahométans menteurs. Chez les écrivains arabes et persans ultérieurs nous n'avons trouvé aucune trace d'un Adam fils de Hōšang.

5. Gayōmart comme père d'Adam et d'Eve. Ce point de vue se trouve dans les textes où l'on dit que Mašyag et Mašyānag sont enfants de Gayōmart et identiques à Adam et $\mathrm{Eve}^{32}$.

Les auteurs de cette conception sont les Persans ${ }^{33}$ ou les Guèbres ${ }^{34}$. Cette dernière information se trouve confirmée dans le livre parsi Huläșah-i Dinn, où l'on dit exactement que Mašyag et Mašyānag (مشي (دشيانه), enfants de Gayōmart, s'appellent Adam et Eve (حوّما وآدم)

Nos premiers rapports de Gayōmart, père d'Adam et Eve, viennent de Bal'amī et Muțahhar ibn Țâhir al-Maqdisĩ dont les œuvres datent de 963 / $964^{36}$ et de $966^{37}$ respectivement. Mais ces deux auteurs ont fait l'usage de sources beaucoup plus anciennes ${ }^{38}$, et l'idée de Gayōmart comme père d'Adam et Eve peut alors aussi être plus ancienne que les dits auteurs.

Ce qui domine dans cette conception n'est pas l'islam mais la religion pré-islamique de la Perse. Même ici c'est Adam qui a du céder la qualité de premier homme à Gayōmart. La cause de l'identification de Mašyag et 
Mašyānag avec Adam et Eve est probablement le fait qu'il s'agit dans les deux cas d'une conception du premier couple humain.

Comme une suite de ce complexe d'identifications nous pouvons regarder ce que Bīrūnī raconte en citant Såìd ibn Muhammad al-Duhlī. On y peut lire que Mašyag et Mašyānag furent élevés dans un endroit qu'on appelle "le paradis » et qu'ils y commirent un péché et puis furent chassés de ce pays $^{39}$. Nous trouvons ainsi ici une partie du récit biblique ${ }^{40}$, mais Mašyag et Mašyānag n'y jouent pourtant pas les rôles des premiers hommes.

6. Gayōmart comme Adam. Cette identification est la mieux attestée. Elle a probablement eu son origine dans le manichéisme, mais nous ne traiterons pas pourtant ici le syncrétisme du manichéisme. La conception de Gayōmart comme Adam appartient aux Persans ${ }^{41}$ et, avant tout, aux mages ${ }^{42}$. C'est ce que disent la plupart des sources, tandis qu'un petit nombre n'en précisent pas les auteurs ${ }^{43}$.

Les auteurs de cette identification sont alors seulement des Persans, pas des Arabes, quoique ceux-ci l'aient pourtant connue. Ceci est un fait très important mais aussi un problème. Nous y reviendrons.

La littérature parsie confirme l'indication des chroniques que les mages sont les auteurs de cette identification. Ainsi une riwāyat parsie fait alternativement usage des noms Gayōmart (كيوسرد) et Adam pour désigner la même personne ${ }^{44}$.

Mais que signifie le mot al-Furs, "les Persans "? Même ceux-ci ont identifié Gayōmart et Adam selon nos sources. Le terme al-Furs comporte une idée nationale tandis que l'expression al-mağüs, "les mages ", concerne la religion. Un mahométan peut être un Persan mais pas un mage. C'est pourquoi nous posons la question : $y-a-t-i l$ eu aussi des mahométans persans qui ont fait l'identification dont il s'agit ici?

Nous avons trouvé un tel mahométan persan, à savoir l'auteur anonyme de Ta’rīh-i Sïstān (de 1060 après J.-C.). Celui-ci dit ainsi : "Gayōmart était Adam, que la paix soit sur lui. $\$^{45} \mathrm{~A}$ cause de cet exemple nous croyons que l'expression al-Furs de nos sources ${ }^{46}$ signifie et mahométans persans et mages, et que certains mahométans persans ont donc aussi identifié Gayōmart et Adam. Mais on ne dit jamais que les Arabes aient fait cette identification, et c'est une chose remarquable. Car les Arabes sont responsables de beaucoup 
des identifications de Gayōmart, et celle avec Adam doit être d'ailleurs la plus proche. Nous en concluons qu'il y a eu un antagonisme entre les Arabes et les Persans au sujet de cette question, et cet antagonisme ne doit pas avoir été religieux seulement mais aussi national.

C'est vrai qu'il y a eu un tel antagonisme selon nos sources. Et on doit le mettre dans un contexte plus grand, à savoir en relation avec les deux

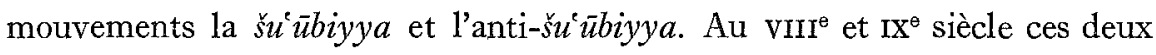
mouvements antagonistes s'intéressèrent aussi de la science généalogique, comme Goldziher l'a démontré47. Mais cet intérêt était en premier lieu conditionné par l'engagement nationaliste des deux mouvements. Ainsi on s'est servi de la généalogie pour glorifier l'origine de son propre peuple (des Persans et des Arabes respectivement) et pour déprécier celui de ses adversaires. - Quand les Persans ont dit que Gayōmart est Adam, voici la tendance de la $\check{s} u$ i übiyya. Et quand les Arabes, les adversaires des Persans, ont dégradé Gayōmart en l'identifiant avec une figure postdiluvienne, voilà la tendance de l'anti-šu' ūbiyya.

Je répète qu'il est vrai qu'on peut discerner un tel antagonisme dans nos sources. Mais cet antagonisme n'est pourtant qu'une continuation d'un antagonisme antérieur, à savoir celui entre les Persans y compris les mages d'un côté et les juifs et les chrétiens de l'autre. On peut le voir très évidemment dans la chronique arabe anonyme nommée Ahlwardt $N^{\circ} 9434$, dont nous avons cité quelques pages dans notre livre sur Gayōmart mais qui n'est d'ailleurs pas encore éditée ${ }^{48}$. Il y a là beaucoup de correspondances verbales avec Tabarī et d'autres chroniqueurs, mais ceux-ci ne sont pas les sources de la chronique anonyme. Car elle s'appuie expressément sur des autorités juives et chrétiennes, et par là nous n'apprenons pas les sources de la chronique anonyme seulement, mais aussi celles de Tabarī et de certains autres historiens.

Selon cette chronique anonyme ce sont des livres juifs et chrétiens qui ont polémisé contre l'identification de Gayōmart avec Adam. On a alors inventé la façon méchante suivante : on a dit, que Gayōmart était la même personne que Gomer, fils de Japhet, fils de Noé, mais que ce Gomer avec le temps est devenu orgueilleux et a alors commandé qu'on le nommât Adam ${ }^{43}$.

Le chroniqueur anonyme mahométan estime beaucoup la solution juive et chrétienne du problème de Gayōmart. Il s'associe de la sorte à cette tra- 
dition antipersane. Et c'est le cas de beaucoup d'autres mahométans aussi, sans que l'on dise que cette tradition soit à l'origine juive et chrétienne.

A cause du mutisme en ce qui concerne l'origine de l'identification de Gayōmart avec Gomer j'ai été de l'avis qu'elle ait été l'invention d'un mahométan arabe jusqu'au moment où je me suis mis au courant de la chronique anonyme. Jusque là je me suis aussi figuré que l'antagonisme (dont Tabarĩ parle) entre les savants des nations au sujet de cette question ait été un antagonisme entre les Persans et les Arabes. Mais maintenant je comprends très bien que Țabarī parle aussi ou peut-être seulement d'un antagonisme entre des Persans d'un côté et des juifs et des chrétiens de l'autre. Tabarī dit ainsi : "Il n'y a pas d'antagonisme entre les savants des nations au sujet de Gayōmart, s'il a été le père des Persans pré-islamiques. Mais ils discutent quant à lui, s'il a été Adam, le père de l'humanité ... ou s'il a été une autre personne. " ${ }^{50}$ Avant ce passage, Ṭabarī fait expressément mention des Persans comme l'une des deux parties combattantes, tandis que leurs adversaires anonymes sont qualifiés comme ceux qui ont identifié Gayōmart avec Gomer ( $\breve{G a} m i r)^{51}$.

Notre preuve la plus ancienne de cette identification se trouve dans le Ta'rīh de Tabari. La date de cette chronique (en 9I2/913 ap. J.-C.) ne peut pourtant pas marquer sa naissance. Au contraire, ce moment doit marquer le début d'une extension amoindrie, car trente ans plus tard Mas'ū $\mathbf{u}$ ĩ atteste que c'est une minorité des Persans qui regarde Gayōmart comme père du genre humain ${ }^{52}$. - Malgré cela Țabarī dit que les Persans ou même que la plupart des savants persans regardent Gayōmart comme l'origine du genre humain ${ }^{53}$. Il y a ainsi une contradiction entre Mas ūî̃ et Țabarī. Cette difficulté disparaît pourtant si l'on comprend les expressions «les Persans » et « la plupart des savants persans " chez Tabarī comme : "la plupart des savants persans dont Ṭabarī connaît les traditions et les livres ». Alors les indications de Tabarī ne concernent pas sa propre époque mais le temps de toutes les sources persanes de Tabarī, celui de la plus ancienne et celui de la plus récente. La plupart de ces sources persanes ont probablement regardé Gayōmart comme Adam.

On peut soutenir la dite interprétation en alléguant la methode de Tabarī. Il rassemble des traditions qu'il a lues ou entendues. Celles-ci tirent son origine de savants du temps islamique ancien ou récent. Dans ce contexte 
faut il comprendre l'indication de Ṭabarī : "La plupart des savants persans disent que Gayōmart est Adam. $y^{54}$

Le débat où les savants persans ont prétendu que Gayōmart soit Adam ne semble pas avoir eu lieu à l'époque de Ṭabarī lui-même à cause d'une autre circonstance : ce débat ne peut pas s'isoler de celui au sujet de Hōšang. Le point de controverse de ce dernier débat a été aussi la question : si un homme persan des temps primitifs a été antédiluvien ou postdiluvien. Et dans les deux cas l'argument des adversaires des Persans est comme suit : "Les Persans ne savent point ce qui était avant Noé. $\$^{55}$ Cet argument se dirige contre chaque conception persane d'une histoire antédiluvienne. Il implique ainsi toujours une dégradation de Gayōmart, même s'il n'est pas lui-même mentionné explicitement.

Le débat en ce qui concerne l'époque de Hōšang a eu lieu vers l'année 800 au plus tard, car Hišām ibn al-Kalbī (m. en 820 ap. J.-C.) l'a raconté selon Țabarī56. Hišām cite l'opinion qu'il a lue ou entendue, mais il semble aussi prendre position lui-même et alors naturellement contre les Persans.

Comme nous l'avons dit, la controverse au sujet de l'époque de Hōšang implique un débat simultané au sujet de celle de Gayōmart. Alors on peut en conclure que celui-ci a eu lieu vers l'année 800 au plus tard.

Cette impression est confirmée par ce qui suit : Dans le rapport de Hišām ibn al-Kalbì on polémise contre les Persans qui "soutiennent qu'il (c.-à-d. Hōšang) vivait deux cents ans après la mort d'Adam 1,57. On dit à peu près la même chose sur la page suivante de Ṭabarī où l'on fait mention de " ceux qui disent que son règne (c.-à-d. celui de Hōšang) arriva deux cents ans après la mort d'Adam ${ }^{58}$. Il est vrai que Hišãm dit à propos du premier passage : «Mais (En réalité), ce roi vivait, selon ce que nous avons appris, deux cents ans après Noé, mais les Persans ont changé cela en deux cents ans après Adam. $1^{59}$ Nous savons pourtant qu'il ne s'agit pas d'une conception originale, ni quand Hōšang figure deux cents ans après Adam ni quand il existe deux cents ans après Noé. Dans les deux cas nous avons une manifestation d'un syncrétisme malgré leurs tendances divergentes. - Mais d'où viennent ces deux cents ans? - Selon nous il n'y a qu'une seule explication de ces deux cents ans : ils représentent l'interrègne entre Gayōmart et Hōšang. En ce qui concerne la durée de cet interrègne il y a des indications différentes ${ }^{60}$, mais la plupart les mentionnent à environ 200 ans. 
Dans le Bundahišn (A, p. $238 ; \mathrm{J}$, p. $8 \mathrm{I}$ ) la durée est de $\mathrm{I} 83^{\frac{1}{2}}$ ans ${ }^{61}$; et selon

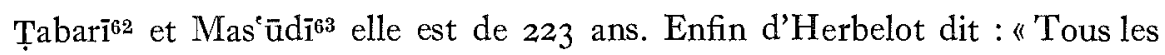
Historiens de Perse marquent un interrègne entre Caiumarath \& lui, qui a duré deux cents ans. ... "64 $^{64}$ Par là nous pouvons conclure comme suit: Si les 200 ans après la mort d'Adam jusqu'au règne de Hōšang dérivent de l'interrègne entre Gayōmart et Hōšang, comme nous le pensons, alors Gayōmart doit avoir été identifié avec Adam par les Persans qui selon Hišām (ou selon les sources de celui-ci) ont dit que la durée entre la mort d'Adam et le règne de Hōšang a été de 200 ans. Par cela même la date la plus ancienne de cette identification serait en environ 800 ap. J.-C. D'ailleurs nous pouvons probablement compter Hišām et ses autorités parmi les représentants de l'anti-šu úbiyya et leurs adversaires persans parmi ceux de la šu übiyya.

On pourrait parvenir à la même date ou peut-être à une date un peu antérieure, si l'on prend certains rapports chez Bal'amì comme point de départ. Nous croyons pourtant que nous avons eu raison d'examiner cette question dans la perspective donnée par TTabarī. Par là nous avons appris les controverses et les tensions liées à cette question.

Les rapports en question chez Bal'amī consistent en une série de noms d'auteurs et de livres qui ont identifié Gayōmart avec Adam. (Voir ci-dessous, p. 287 n. 4r.) Ce sont pour la plupart des traducteurs, des compilateurs et des rédacteurs de différentes versions arabes du livre royal sassanide ${ }^{65}$. Nous ne pouvons pas déterminer leur temps exact, mais certains en ont probablement écrit pendant la dernière moitié du viII ${ }^{\mathrm{e}}$ siècle (en tous cas après le livre royal d'Ibn al-Muqaffa'), d'autres pendant le $\mathrm{IX}^{\mathrm{e}}$ siècle. Ces auteurs ont toutes les qualités requises pour constituer une partie du groupe que Tabarī nomme "la plupart des savants persans ", c.-à-d. du groupe qui selon Tabarī identifie Gayōmart avec Adam. Certains de ceux-ci ont probablement aussi pris part au débat si le père des Persans a été antédiluvien ou postdiluvien, à celui dont Ṭabarī parle d'après Hišām ibn al-Kalbī et qui doit alors avoir eu lieu en 800 environ au plus tard.

Nous avons adopté la classification du baron Rosen et de Christensen ${ }^{66}$ en ce qui concerne les auteurs persans qui selon Bal'amī ont identifié Gayōmart avec Adam. Les voici : 
A. Les traducteurs :

I. Muhammad ibn al-Ğahm al-Barmakī

2. Zādūyah ibn Šāhūyah al-Ișfahānī

B. Les traducteurs-compilateurs :

r. Hišām ibn Qāsim al-Iṣfahānī

C. Les "rédacteurs》:

r. Mūsa ibn 'Īsā al-Kisrawī (dont la chronique date d'environ 860)

2. Bahrām ibn Mardānšāh, mobad de la ville Šāpūr

D. D'autres :

r. Ardavad Murḡān, mobad des mobad.

Cette série de personnes contient et des mahométans et des mages ce qui convient à l'image que Țabarī nous a donné déjà.

Ci-dessus nous avons dit que l'on ne peut pas croire que « la plupart des savants persans " d'environ goo aient identifié Gayōmart avec Adam. Mais avant cette date l'identification en question a certainement été en vogue longtemps en Perse. Son influence doit pourtant avoir cédé peu à peu jusqu'à environ $94^{\circ}$ où elle a été mince. Ce changement est attesté par Mas' ${ }^{e} \mathrm{dĭ}^{67}$ comme nous avons vu, et il doit s'expliquer par le fait que la polémique contre la šu $u^{e} u \overline{b i y y a}$ culmina pendant le $\mathrm{IX}^{\mathrm{e}}$ siècle ${ }^{68}$, et que la šu übiyya extrémiste disparait ensuite.

Le changement en importance de cette identification se montre dans l'attitude des auteurs persans après l'année 950. Nous n'en avons trouvé qu'un seul parmi une trentaine, qui a fait cette identification lui-même, et celui-ci est l'auteur susmentionné de Ta'rīh-i Sīstān (aux environs de Io6o) ${ }^{69}$. Et ce qui est vraiment notable c'est ce que nos sources persanes d'après l'année IIoO, attribuent cette identification aux «mages 》 ou aux guèbres ${ }^{70}$.

Conclusion : l'identification de Gayōmart avec Adam, à l'époque islamique, se trouve d'abord chez les mages mais aussi chez les mahométans persans. Peu à peu elle a perdu du terrain parmi ceux-ci et enfin n'a existé que parmi les mages, c.-à-d. au dehors de l'islam.

Après cette conclusion il faut admettre que nous avons caché certains rapports qui la contredisent. Ceux-ci se trouvent chez les auteurs arabes

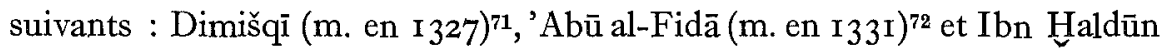
(m. en $\left.{ }^{4} 406\right)^{73}$. Ces trois Arabes déclarent que les Persans identifient GayōI $8-68438$ I Hartman 
mart avec Adam. Ibn Haldūn dit même que ce sont "tous les Persans » qui font ainsi ${ }^{74}$. Nous ne pouvons pas pourtant regarder ces rapports comme valables au temps propre de ces auteurs. Car ils sont en contradiction avec tous les auteurs persans du même temps ${ }^{75}$, et les rapports de ceux-ci sont plus valables dans ce cas. Les rapports des dits auteurs arabes dépendent certainement de sources relatives au temps d'avant Mas'ūìi.

La cause du fait qu'on a identifié Gayōmart avec Adam est évidente : tous les deux ont été liés à la conception du premier homme. - Mais pourquoi les Arabes n'ont ils pas accepté leur identité? - La cause en est la suivante : les Arabes ont estimé les différences entre les deux personnes plus importantes que les ressemblances. Nous allons citer quelques arguments, que les Arabes ont allégués dans ce contexte. Les Arabes sont alors certainement dépendants des juifs et des chrétiens.

Hišām ibn al-Kalbī a selon Ṭabarī repoussé la prétention des Persans que le roi Hōšang ait vécu 200 ans après Adam (qui alors serait le même que Gayōmart ${ }^{76}$ ) en disant : «Ils (c.-à-d. les Persans) ne savent point ce qui était avant Noé. $\$^{77}$ - Celle-ci est lobjection la plus importante, et elle revient çà et là dans la littérature ultériqure. Un autre passage chez Țabarī souligne son importance : "Quant aux mages, ils ne connaissent pas le déluge, parce qu'ils disent : 'La royauté n'a pas cessé chez nous depuis l'époque de Gayōmart.' Et malgré cela ils disent : 'Gayōmart est Adam.' ')78 Il y a ici un contraste entre le prétention des Persans que Gayōmart soit Adam et leur ignorance du déluge. Et la tension augmente à cause de l'allégation des Persans que depuis Gayōmart il y ait toujours eu des rois persans. Car alors faut-il naturellement connaitre le déluge selon la conception islamique (et juive et chrétienne). - Telles sont les difficultés qui expliquent pourquoi les Arabes ont nié l'identité de Gayōmart et Adam.

Mais certains Persans ne se sont pas résignés à cause de l'argument du déluge. Ils ont trouvé une défense pour leur histoire d'origine. Ils ont dit, en effet, qu'ils ont connu le déluge mais que celui-ci n'a pas été universel. Il n'a touché que le climat (اقليم) de Babel et ses environs, tandis que les descendants de Gayōmart ont habité l'Orient où ils ont été hors de danger. C'est pourquoi les rois persans ont eu une succession ininterrompue depuis Gayōmart qui a aussi pu vivre avant le déluge. Ceci est le rapport de Tabarī79 et il peut se compléter par Bīrūnī qui dit que ce déluge partiel aurait eu lieu 
à l'époque de Taḩmōruf ${ }^{80}$. Et, comme nous avons déjà vu, même Hamzah décrit un déluge à l'époque de Taḩmōruf, et ce déluge est aussi partiel n'ayant frappé que l'Occident ${ }^{81}$. - Il s'agit ainsi de la même sorte de déluge chez Ṭabarī, Bīrūnī et Ḥamzah. Par là nous savons que la défense des Persans contre les Arabes (et peut-être aussi contre les juifs et les chrétiens) tire son origine en dernier lieu du récit de Bérose sur Sisouthros, car nous avons vu ci-dessus que c'est le cas pour le déluge de Hamzah ${ }^{82}$. Le fait d'être partiel est un trait que le déluge a reçu probablement sous l'influence du christianisme syriaque, qui a d'ailleurs procuré les traditions de Bérose ${ }^{83}$.

Contre l'allégation des Persans que le déluge n'ait pas été universel et n'ait pas touché les descendants de Gayōmart Țabarī ${ }^{84}$ (et après lui d'autres auteurs $^{85}$ ) cite le Coran XXXVII, 73-75, où l'on peut lire qu'Allāh sauva Noé et sa famille. Et le paragraphe qui dit : «Et nous avons fait survivre sa postérité "86 est commenté ainsi : "Et il (c.-à-d. Allāh) ... raconte, que la postérité de Noé furent les seuls survivants. "87 Même cette interprétation se trouve dans des œuvres plus récentes ${ }^{88}$.

Mais on a eu aussi d'autres arguments contre l'identification de Gayōmart avec Adam. Táālibì dit comme suit : "Mais comment cette identité (litt. cela) serait elle admissible, puisqu'on dit dans les chroniques qu'Adam que la paix soit sur lui - après sa descente sur la terre vécut mille ans, tandis que Gayōmart ne régna que trente ans? "19 Cette objection ne peut pas avoir été forte partout et toujours, car plusieurs sources nous montrent qu'on a aussi attribué à Gayōmart une vie de mille ans ${ }^{90}$, quoique cette affirmation sur Gayōmart soit certainement secondaire et dépende de son identification avec Adam ${ }^{91}$. Selon une tradition ancienne islamique la vie d'Adam aurait duré mille ans ${ }^{92}$.

Par ce que nous venons de dire nous avons fait mention d'une des conséquences de cette identification. Il y en a d'autres, et elles ont affaire et au caractère de Gayōmart et à celui d'Adam. En voici quelques exemples.

Táāâlibī dit ce qui suit : "Et certains d'eux (c.-à-d. des chroniqueurs) disent qu'il est le même qu'Adam, le père du genre humain, qu'Allāh - il est élevé - a créé de sa main, en qui il a insufflé (une parcelle) de son esprit, qu'il a fait adorer par tous ses anges.... $\rangle^{93}$ - Les dits détails appartiennent d'ordinaire à Adam, et il est certainement inutile de le démontrer ici. C'est de même avec ce que dit Šukr Allāh, à savoir : "Certains des non- 
Arabes disent que Gayōmart, exactement comme Adam, le choisi (d'Allāh), fut créé de la poussière, sans père et mère. 194

7. Gayōmart comme un fils d'Adam, mais pas Caïn, Abel ou Seth. Dans cette partie je traite les rapports où Gayōmart figure comme un fils d'Adam $^{95}$, un frère de Seth ${ }^{96}$ et le fils le plus âgé d'Adam ${ }^{97}$. C'est très vraisemblable que ces trois expressions reflètent une seule conception. Car quand Gayōmart est qualifié d'un fils d'Adam, il fait la guerre contre les enfants de Cain-Qābīl selon certaines sources ${ }^{98}$, et selon un auteur cette guerre a lieu sur l'ordre de Seth" ${ }^{99}$. Alors c'est impossible que ce Gayōmart, fils d'Adam, soit lui-même Seth ou Caïn, mais c'est nécessaire qu'il soit un de leurs frères, et alors c'est très probable que les deux expressions «fils d'Adam 》 et "frère de Seth » se rapportent à la même personne. - Au sujet des deux expressions "frère de Seth 》 et "le fils le plus âgé d'Adam "leur valeur égale apparaît du fait que Ġazzālì a nommé Gayōmart et le frère de Seth ${ }^{100}$ et le fils le plus âgé d'Adam ${ }^{101}$.

Par ce que nous venons de dire nous avons voulu motiver pourquoi nous avons traité ici comme une seule personne le Gayōmart, fils d'Adam, le Gayōmart, frère de Seth, et le Gayōmart, le fils le plus âgé d'Adam.

L'expression "le fils le plus âgé d'Adam " nous semble demander une explication de plus. Si l'on compare les sources sur lui, on peut constater qu'il s'agit de celui des fils d'Adam qui a vécu le plus longtemps, celui qui est resté après la mort de tous les autres fils d'Adam ${ }^{102}$. Il ne s'agit pas alors du premier-né des fils d'Adam.

Le trait le plus essentiel de ce fils le plus âgé d'Adam est sa royauté : il a été le premier roi du monde, ou au moins le premier roi doué d'insignes royaux.

Qui peut il bien être, ce fils le plus âgé d'Adam? Pour répondre à cette question il faut considérer un peu les circonstances de cette figure.

Quand on nomme Gayōmart tout vaguement fils d'Adam, cela ne signifie pas absolument qu'il est identifié avec un certain fils sur lequel il y a déjà beaucoup d'idées dans l'islam. Mais cela signifie nécessairement que Gayōmart est mis en relation avec Adam et avec les descendants d'Adam, ce qui n'a pas été le cas plus tôt.

Mais dans certaines sources il y a de telles informations que nous compre- 
nons que Gayōmart, fils d'Adam, a été identifié avec une certaine personne, au moins parfois. (Cette certaine personne n'est pourtant pas d'ailleurs spécialement connue comme fils d'Adam, et c'est probablement pourquoi nos sources s'expriment assez vaguement.) Cela arrive quand on dit que Gayōmart, fils d'Adam, conduit les enfants de Seth dans leur guerre contre les enfants de Cain. Cet épisode se trouve dans les ouvres de certains auteurs qui regardent eux-mêmes Gayōmart comme un fils d'Adam ${ }^{103}$ et dans quelques livres où l'on rapporte seulement cette opinion ${ }^{104}$. (Les auteurs qui traitent eux-mêmes Gayōmart en tant qu'une figure pré-adamite ou postdiluvienne n'ont naturellement pas pu décrire sa guerre contre les enfants de Caïn-Qābīl.) A cause de ce que cette guerre selon certaines traditions est une caractéristique des rois chaldéens antédiluviens ${ }^{105}$, dont le premier a été identifié avec Gayōmart ${ }^{106}$, comme nous l'avons vu, à cause de cela nous comprenons très bien que Gayōmart, fils d'Adam, a été le même qu'Aloros, à savoir, pour ceux qui racontent la guerre de ce Gayōmart contre les enfants de Caïn-Qābĩl. Et le dit Aloros convient aussi très bien en tant que "le fils le plus âgé d'Adam " dans le sens déterminé ci-dessus, c.-à-d. en tant que celui des fils d'Adam qui a vécu le plus longtemps, car il régnait selon Bīrūnīior entre l'année Io64 et l'année I I62 après la création.

Mais il n'y a pas un seul livre que je sache où l'on dit qu'Aloros a été regardé comme un fils d'Adam. Nous savons seulement ce qui suit : Aloros et ses neuf successeurs ont été interpolés dans l'histoire biblique avant le déluge par des chrétiens grecs, syriens et arméniens de même que par des écrivains mahométans, et tous ceux-ci ont - directement ou indirectementidentifié le dernier des chaldéens antédiluviens, à savoir Sisouthros, avec Noé108. Mais les rapports de ces sources présupposent dans ce cas qu'il y a eu un lien de parenté entre Adam et Aloros, un lien qui n'est pourtant pas précisé comme celui entre Adam et Gayōmart quand celui-ci doué des propriétés d'Aloros est nommé fils d'Adam. - Si Aloros n'est pas expressément dit fils d'Adam, ceci ne contredit donc pas nécessairement la supposition vraisemblable que Gayōmart en tant que fils d'Adam a été conçu comme identique à Aloros.

Voici maintenant une liste de ceux qui se sont rangés à l'avis que Gayōmart est un fils d'Adam : 
"Certains 》 (des historiens) ${ }^{109}$,

"Les Persans "110,

"Certains » (des Persans ou des savants persans)"11,

"Les généalogistes des non-Arabes et des Persans "112,

Certains auteurs persans individuels : Ġazzālī113, 'Aufīi114, Harawīi115, Šabānkāra' î116, Zahīr al-Dīnn'117, Kūhistānī118, 'Aḥmad ibn Bahbal119 . — Parmi ceuxci il faut noter spécialement Ġazzālī, le grand mystique et théologien islamique. Son autorité citée dans plusieurs livres a certainement fait beaucoup pour l'extension de cette identification.

Cette liste est d'un intérêt particulier. Car elle ne contient que des Persans, et naturellement des Persans mahométans. Aucun Arabe! Cela signifie que le Coran a été la norme pour ces Persans quand il s'est agi de déterminer qui a été le premier homme. Mais ils ont aussi voulu défendre leurs prétentions nationalistes : car Gayōmart est devenu le premier roi du monde, et même un roi antédiluvien. On a ainsi repoussé la déclaration railleuse que les Persans soient ignorants au sujet de l'époque d'avant le déluge. Certains de ces auteurs semblent même défendre ou du moins expliquer l'origine de la conception que Gayōmart soit le premier homme. 'Aufī (en I228) dit par exemple que l'on a appelé Gayōmart " le deuxième Adam à cause de ce qu'il a été brun foncé et a eu une beauté tellement digne que chacun qui le voyait se prosternait (devant lui). $\$^{20}$

Au sujet de la date d'origine de la tradition sur Gayōmart, fils d'Adam, notre source la plus ancienne est la chronique de Tabarī (d'environ 900 ap. J.-C.). Mais Ṭabarī lui-même s'autorise dans ce contexte de savants persans, et c'est pourquoi nous avons raison de nous représenter que la tradition en question est plus ancienne.

Quel a été le point de contact entre Gayōmart et le fils d'Adam en question? - Si ce fils d'Adam a été à l'origine le même qu'Ailoros, le point de contact a été la propriété de tous les deux d'être le premier roi du monde. D'ailleurs ces deux personnes appartiennent au temps primitif, mais ce fait seul pourrait difficilement produire l'identification en question.

Les suites de cette identification sont les contes sur Gayōmart comme guerrier parmi les séthites contre les caïnites, dont parlent aussi certaines sources chrétiennes et islamiques. 
8. Gayōmart comme Seth. Deux livres disent que Gayōmart est le même que Seth, fils d'Adam, selon " un peuple "121 ou selon «les grecs »122. La chronique anonyme parle de livres grecs où l'on fait mention de Gayōmart, c'est vrai. Mais le Gayōmart de ces livres est le même que Gomer, le petit-fils de Noé. Peut-être y-a-t-il eu d'autres livres grecs où notre héros a figuré. Dans ce cas ils ont certainement aussi été chrétiens.

9. Gayōmart comme un fils de Caïn-Qäbïl. La seule source de cette tradition est Sukr Allāh (dont le livre date de 1456). Il rapporte d'abord la conception arabe et dit alors que certains prononcent le nom : ك̆ayūmart, c.-à-d. avec la lettre ğ $̌ m m$ au début, et que ceux-ci " disent après cela qu'il est (un) des enfants de Caīn (Qābil) »123. Cette petite notice est la seule que nous ayons à notre disposition. Elle nous apprend ce qui suit : les inventeurs de l'identification sont des Arabes, ennemis des Persans. Leur argumentation a peut-être été comme suit : les enfants de Cain-Qābīl ont été des apostats à qui 'Iblis avait enseigné l'adoration du feu et qui avaient fait des temples de feu ${ }^{124}$. C'est pourquoi Gayōmart a du appartenir à ceux-ci, s'il a absolument vécu avant le déluge. Et l'adoration du feu est naturellement le point de contact entre les Persans et les enfants de Caïn-Qābìl. - Malgré a date tardive de la source de cette tradition elle pourrait dériver des premiers siècles de l'islam, où l'anti-šu'übiyya était très forte.

I0. Gayōmart comme un fils de Seth. Sukr Allāh est notre seule source de cette identification, et il dit que son origine est chez " certains des Persans 》 (بعضعجم) (بعient très bien, car il s'agit vraiment d'une identification positive (le lignage de Seth et l'époque antédiluvienne). C'est tout ce que nous pouvons dire.

II. Gayōmart comme Kénan. Cette identification est attribuée aux Persans ${ }^{126}$. Elle est combinée aux récits sur les guerres entre les descendants d'Adam et à d'autres des traditions au sujet de l'histoire antédiluvienne. Il y a aussi beaucoup de matériaux non-bibliques au sujet de Gayōmart-Kénan. Grâce à la traduction d'un manuscrit de la Bibliothèque Nationale nous savons que c'est Ibn al-Muqaffa' (m. en 757 ap. J.-C.) qui a raconté l'histoire de Gayōmart-Kénann ${ }^{127}$. Par là cette identification est celle datée le plus tôt. 
(Plusieurs autres identifications sont certainement d'une date antérieure mais la documentation en fait défaut.)

I2. Gayōmart comme un fils de Mahalalee1. Ceci est une croyance chez les Persans selon d'Herbelot ${ }^{128}$. On la trouve même dans quelques œuvres persanes ${ }^{129}$, mais on ne nous en donne aucuns détails ultérieurs. Date d'origine: avant la chronique de Bal amī (de 963 ap. J.-C.).

3. Gayōmart comme un petit-fils de Mahalaleel. Cette identification ne se trouve que dans quelques œuvres persanes très récentes ${ }^{130}$. Il s'agit en tous cas d'une opinion assez positive sur Gayōmart : il appartient aux descendants de Seth et à l'époque antédiluvienne.

I4. Gayōmart comme Noé. Il n'y a qu'une seule source (de I5I I) qui en fait mention ${ }^{131}$. Pas de détails ultérieurs.

Avant de commencer le développement des identifications postdiluviennes il faut noter quelque chose au sujet des identifications antédiluviennes : Il n'y a que deux de celles-ci qui ont leur origine chez les Arabes : les identifications péjoratives avec un ğinn et avec un fils de Caīn-Qābīl. Ceci dépend naturellement de l'argumentation des Arabes, empruntée des juifs et des chrétiens, citée ci-dessus. (Voir p. 269.) - Il faut même remarquer la prédilection des Persans pour les descendants de Seth. Ceci implique une succession ininterrompue à partir de Gayōmart et indépendamment du déluge pour les rois des Persans.

I5. Gayōmart comme un fils anonyme de Noé ou vraiment comme Sem. Le représentant le plus important de cette identification est Baiḍāwī (m. en I292/I 293 ap. J.-C.). Ce savant a réfléchi beaucoup sur tout le problème, et son argumentation revient chez plusieurs auteurs ultérieurs même quand il s'agit de d'autres identifications postdiluviennes. Il dit comme suit : «Et beaucoup de gens disent, qu'il est un des enfants de Noé - que la paix soit sur lui. Et cela est plus évident à cause de ce qu'on est d'accord sur le fait qu'Abraham, l'ami sincère, - que la paix soit sur lui - a existé au temps de Ḍaḥhāk; et depuis les jours de Ḍaḥhāk jusqu'à l'époque de Gayōmart il y a eu mille ans, et depuis le temps d'Abraham jusqu'au temps du déluge il y a 
eu presque mille quatre cents ans; et de la même manière on s'est mis d'accord sur le fait que Moïse - que la paix soit sur lui — a existé au temps de Minūčihr; et depuis son temps jusqu'au temps de Gayōmart il y a presque deux mille deux cents ans selon ce que disent les Persans; et, selon ce que disent les savants des enfants d'Israël, il y aurait presque cet espace de temps depuis les jours de Moïse jusqu'au temps du déluge. Troisièmement : les généalogistes de Perse donnent à Ḍaḥāk par (l'intermédiaire de) trois pères une origine en Tāa, qui était le père des Arabes et le frère de Hōšang, qui faisait partie des petits-fils de Gayōmart, qui est la racine des rois de Perse; et les généalogistes des Arabes donnent (à Dahḥāk) par (l’intermédiaire de) trois pères une origine en Aram parmi les enfants de Sem, fils de Noé, (Aram) qui était le père des Arabes et le frère d'Arpacschad, qui était le père des Persans. Et l'accord entre ces deux récits est sur le fait que Tāz est Aram; et Hōšang, Arpacschad; et Gayōmart, Sem, fils de Noé. „132

L'argumentation de Baiḍāwĩ est assez faible, car elle présuppose qu'il y ait certaines identifications et adaptations infaillibles entre des conceptions arabes et des conceptions persanes, et qu'on puisse en faire usage comme des points de départ. Ainsi elle préconise néanmoins qu'il soit certain qu'Aždahāk soit le même et que Ḍaḥ̂āk et que Nimrod, et qu'il ait vécu à l'époque d'Abraham, et que Minūčihr ait vécu à l'époque de Moïse. - Mais pour nous l'argumentation en question est d'un grand intérêt, car elle cherche évidemment à unir les désirs des Persans et ceux des Arabes, mais cela s'est fait pourtant aux dépens de ceux-là.

I6. Gayömart comme un fils de Sem. Ce sont des historiens persans ${ }^{133}$ tardifs qui présentent cette opinion. Que l'on ait placé Gayōmart après le déluge, ceci dépend certainement des arguments que les Arabes ont emprunté des juifs et des chrétiens et de ceux de Baidāwī de ci-dessus. Sa qualité d'être un fils de Sem contient parfois une polémique contre la conception qu'il soit un descendant de Japhet. Ainsi Mìr $\mathrm{H}^{\mathrm{w}} a \bar{n}$ d l'identifie avec un fils de Sem et dit à la fois que la conception qu'il soit un Japhetite " contredit ce que tous les historiens disent, car leur opinion est celle que tous les Hān du Turkestan appartiennent aux enfants de Japhet mais pas les rois (les $p \bar{a} d \bar{s} \bar{a} h)$ de Quraiš, parce que tous ayant ce rang appartiennent aux descendants de Gayōmart. ( ${ }^{\mathbf{1 3 4}}$ - Dans cette argumentation on présente 19-68438r Hartman 
deux propositions en tant qu'axiomes : a) tous rois descendent de Gayōmart ${ }^{135}$; b) les rois de Quraiš ne descendent pas de Japhet (mais de Sem). A cause de ces deux axiomes Gayōmart ne peut pas descendre de Japhet mais du même ancêtre que Quraiš, à savoir de Sem.

Cette identification attestée tardivement a son origine chez des mahométans persans. Elle accepte évidemment le caractère postdiluvien de Gayōmart, mais elle proteste contre une dégradation trop radicale. Gayōmart reste en tous cas l'ancêtre de tous les rois du lignage de Sem. Et par là les Persans peuvent malgré tout réclamer une origine plus noble que les Arabes par exemple. L'identification de Gayōmart avec le fils anonyme de Sem révèle ainsi, et une bonne foi islamique, et un bon nationalisme persan.

I7. Gayōmart comme Gomer, fils de Japhet, fils de Noé. Nous avons déjà ci-dessus à propos de l'identification de Gayōmart et Adam fait remarquer que l'identification avec Gomer a été le contre-coup le plus fort contre celle-là, et que cette dernière identification tire son origine de chroniques juives et chrétiennes et puis a été adoptée par des mahométans arabes ${ }^{136}$. C'est notre supposition que l'identification en question ait été adoptée par des Arabes, mais c'est une supposition nécessaire, semble-t-il. Car la tradition sur Gayōmart-Gomer se présente assez souvent comme une critique des Persans et de leurs prétentions au sujet de l'origine de leurs rois. Et il ne s'agit pas alors des mages seulement mais aussi des mahométans persans. Par là ce débat pourrait refléter la controverse entre la šsu'übiyya et l'antišu'übiyya.

Il faut pourtant immédiatement admettre que Țabarī dans un passage ${ }^{137}$ parle de ce que certains savants persans ont dit en ce qui concerne Gomer, fils de Japhet, fils de Noé, et que ce Gomer alors reçoit l'épithète «le père des Persans „138 et une vie de mille ans. Ce Gomer a naturellement été identifié avec Gayōmart et alors reçu ces qualités. Et la vie de mille ans dépend certainement du fait que ce Gayōmart a plus tôt été identifié avec Adam ${ }^{139}$. Même Ibn Ḩaldūn attribue cette identification aux Persans. Il dit : «Certains des savants persans disent, que Gayömart est le même que Gomer (Kümar), fils de Japhet, fils de Noé. „140 Mais cet auteur a sur la page précédente dit : " tous les Persans sont d'accord sur le fait que Gayōmart est Adam, "141 
On peut discerner deux causes primaires de cette identification :

a) l'argument que les Persans n'ont pas connu le déluge et que leur histoire d'origine est ainsi postdiluvienne;

b) la ressemblance entre les deux noms Gayōmart et Gomer ${ }^{142}$.

Cette identification tire peut-être son origine de l'époque préislamique. Mais elle doit en tous cas être plus récente que celle avec Adam, parce qu'elle se rapporte à un récit polémique contre celle-ci. (A savoir le récit sur le Gomer orgueilleux qui se nomma Adam.)

Parmi les suites de cette identification on note les suivantes : on dit qu'il a colonisé l'Orient et qu'il a reçu comme des hôtes son frère Madaï, fils de Japhet, fils de Noé, et son oncle Elam, fils de Sem. Celui-là (à savoir Madaï) a plus tard, dit on, été roi d'une partie du royaume de Gayōmart, et il y a été très puissant. On en dit autant d'Elam ${ }^{143}$. Il s'agit ainsi des récits bibliques sur la Médie et l'Elam réunis ici avec la figure de Gayōmart.

\section{I8. Gayōmart en tant que 'Umaim, filı de Lāwad, fills d'Aram, fils de} Sem, fils de Noé. Les auteurs de cette identification sont selon Mas'ūdī « un groupe d'entre eux » (c.-à-d. d'entre les Persans)144. Mais chez Ğūzğānīit4 il semble qu'il s'agit ici d'une identification de provenance arabe, car nous y lisons : "Certains ont dit : elle (c.-à-d. sa généalogie) appartient aux généalogies des Arabes, c.-à-d. il était un des enfants de Sem, fils de Noé, et son nom était 'Umaim, fils de Lāwad, fils de Sem, fils de Noé. "Bīrūnī fait le contraste entre cette identification (avec le récit sur Gomer) et l'attitude des Persans et des Mages envers le déluge et leurs prétentions de connaître l'histoire originale de l'humanité.

Les dites sources indiquent ainsi une provenance arabe de l'identification de Gayōmart avec 'Umaim. Ibn Qutaiba (m. en 889) les appuie : après avoir traité trois fils de Lāwad, il dit ainsi : «Et leur frère 'Umaim, fils de Lāwad, fils d'Aram, fils de Sem, fils de Noé, s'installa en Perse, et toutes les familles des Persans descendent de ses enfants. $\rangle^{146}$ Cette information n'est pas unique ${ }^{147}$, c'est vrai, mais ce qui est remarquable c'est le fait qu'elle se trouve dans un livre d'Ibn Qutaiba précisément. Car cet écrivain a certaines qualités qui ont de l'importance dans notre contexte : a) Il était lui-même persan et connaissait très bien des traditions persanes, et il cite parfois même la traduction arabe du livre royal sassanide faite par Ibn al-Muqaffa ${ }^{2}{ }^{148}$. b) Malgré cela il 
était un ardent adversaire de la šúübiyya et par là un ennemi des prétentions des Persans si celles-ci entraient en collision avec celles des Arabes ${ }^{149}$. C'est ce Ibn Qutaiba qui d'après des traditions arabes décrit comment la terre fut peuplée après le déluge, comment alors Sem s'installa au centre de la terre sacrée, c.-à-d. à La Mecque et Médine et leurs environs ${ }^{150}$. C'est le même Ibn Qutaiba qui raconte, après cela, que la Perse a été peuplée d'abord par 'Umaim, fils de Sem, quoiqu'il sache très bien les traditions de ses compatriotes sur leur histoire d'origine. Cette représentation d'Ibn Qutaiba implique peut-être déjà l'identification de Gayōmart avec 'Umaim ou avec un descendant de celui-ci, spécialement à cause de sa déclaration que toutes les familles persanes sont issues de 'Umaim. Mais si l'identification en question n'est pas impliquée dans ce que dit Ibn Qutaiba, son récit doit en avoir été la condition.

Conclusion : L'idée d'une provenance des Persans chez 'Umaim a appartenu aux groupes amis des Arabes et amis de l'anti-šs'übiyya. Parce que c'est cette idée qui a inspiré l'identification de Gayōmart avec 'Umaim, celle-ci doit avoir son origine dans les mêmes groupes.

Mas'ūdì nous aide à fixer la date d'origine de l'idée de 'Umaim comme l'ancêtre des Persans : elle s'est trouvée déjà à la première époque de l'islam. Car il en cite un poème dont l'auteur est " quelqu'un parmi les premiers sages des poètes persans après l'apparition de l'islam. "151

Quand on attribue cette identification même aux Persans, cela s'explique probablement par le fait qu'il y avait aussi des Persans dans le mouvement le l'anti-šu'übiyya. (Ibn Qutaiba n'en est qu'un exemple.) Elle n'a jamais pourtant eu de l'importance, semble-t-il, parmi les Persans.

Comment expliquer enfin la différence des deux noms Gayōmart et 'Umaim? Deux sources récentes nous en donnent la solution suivante : 'Umaim a reçu le nom Gayōmart comme laqab, "surnom », au début de son règne ${ }^{152}$.

Ig. Gayōmart en tant qu'un fils de 'Ưmaim. Cette identification dépend naturellement de l'idée arabe traitée tout à l'heure que 'Umaim a été l'ancêtre des Persans. Elle se trouve d'abord chez Mas'üdì153 mais peut naturellement être d'une date antérieure. 
Nous avons traité ici 19 des identifications de Gayōmart. Par là nous avons eu affaire à 19 traditions différentes sur lui. Chacune de ces traditions se trouve cependant en contexte avec le reste de l'histoire persane. Pour obtenir 19 représentations de toute l'histoire légendaire des Persans nous devrions ainsi examiner les traditions et les identifications islamiques des autres figures persanes et puis unir les traditions d'une même origine. Naturellement nous ne pouvons pas réaliser un tel projet complètement, mais en tous cas nous pourrions probablement arriver à dessiner les contours de ces I9 systèmes différents.

\section{NOTES}

1 Cela s'ensuit de la littérature pehlevie, le Bundahišn, éd. Anklesaria, pp. 24 et IOO, p. ex.

$2 \mathrm{I}$, pp. $87 \mathrm{~s}$.

3 Bibliothèque Orientale (Nouvelle édition 178 I-I783) II, pp. I78 s.

4 Kitäb al-Muhabbar (éd. Lichtenstädter, Hyderabad I36r/1942), p. 392 (au sujet des rois du monde) : اول من ملكها من ولد الجان جيوهرت

5 Au sujet de cet auteur et son œuvre, voir Rieu, Catalogue of the Persian Manuscripts in the British Museum III, p. 884, et Storey, Persian Literature II, pp. 9r s.

- Voir mon livre Gayōmart, pp. 202 et LXII :

7 Cet ordre est remarquable mais ne dépend pas nécessairement d'une tradition erronée, car même " le livre royal 》 des mandéens faisant partie du Sidrā Rabbā place Taḩmōruf immédiatement après Gayōmart. Mais Hōšang n'y figure pas du tout. Voir Zeitschrift für Assyriologie 19, I905-1906, p. 74 (transcription et traduction par Sch. Ochser), et cf. pp. $274 \mathrm{~s}$. (commentaires par Louis H. Gray). L'ordre habituel est ainsi : Gayōmart, Hōšang, Tahymōruf. Voir Christensen I, pp. I29 s.

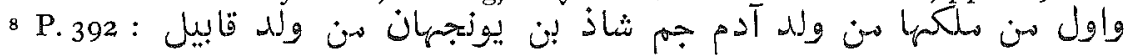

9 Bundahišn A, p. 24: šašom martōm dät ku gayōmart, "sixièmement : Il créa l'homme, c.-à-d. Gayōmart. " Cf. aussi Bundahišn A, p. Ioo.

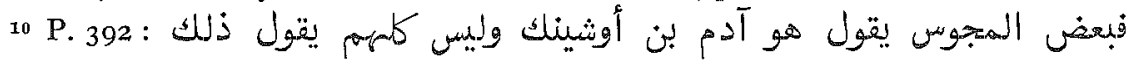

11 Bibliothèque Orientale III, pp. $33 \mathrm{~s}$.

12 Voir mon livre Gayōmart, pp. 202 et LxIr.

19 Ibidem.

${ }^{14}$ Ed. Cureton, p. 182.

${ }_{15}$ Voir mon livre Gayōmart, pp. 148 et xxv.

16 Ğūzğănī, Tabaqqāt-i Nãsisiñ. Voir mon Gayōmart, pp. I5I ss. et XxvII ss. - Au sujet de l'auteur et son œuvre, voir Storey II, pp. 68 ss. et Rieu I, pp. 7 I ss. 
17 Georgius Syncellus, éd. Dindorf, p. 7 I : 3 s. Eusebius, Chronicorum liber prior,

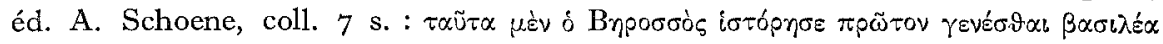

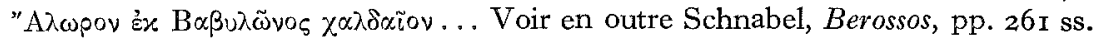

18 Dhorme, "L'aurore de l'histoire babylonienne ", Revue biblique, sér. I, 33, r924,

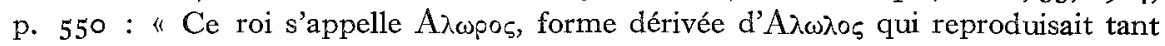
bien que mal Alulim, la terminaison babylonienne étant remplacée par une terminaison grecque."

19 Voir mon article "Die vorflutlichen Chaldäer in arabischer Überlieferung ", Orientalia Suecana, 14-15, 1965-1966, pp. 89-98.

20 Voir Gayōmart, pp. 154 et XxIX s.

21 Storey II, pp. 68 s., Rieu I, pp. 72 s.

22 Cette façon d'écrire le nom se trouve chez Georgius Syncellus, p. 69 (Eusèbe, col. 32) et doit être plus originale que les autres. Dhorme dit : "Il est maintenant évident que le nom sumérien $Z i-u d-s u d-d u$ a pu donner naissance à $\Sigma$ ioovipos (d'où Eเซov 9 pos), d'autant plus facilement qu'on a la forme abrégée $Z i$-sud-da dans un vocabulaire. " Revue biblique, sér. I, 33, r924, p. 552 .

${ }_{23}$ Zoroastrische Studien, pp. $208 \mathrm{~s}$.

24 Op. cit., p. 209.

${ }_{25}$ Hamzah al-Ișfahäni, Ta'rīh mulük al-'ard, éd. Calcutta, I 822, pp. I72 ss.; éd. Gottwaldt, 1844, pp. 197 ss., trad. pp. I5I ss. Trad. en partie même chez Christensen I, pp. I96 ss.

26 Enz. d. Islam, I.

${ }_{27}$ Cette conception se trouve ci-dessus dans ce que nous avons cité de Ğūzgăni. Même Mas'ūdī la témoigne dans son livre Kitāb al-tanbīh wa al-'ǐšrāf, Bibliotheca Geographorum Arabicorum VIII, p. 94 (trad. par Carra de Vaux sous le titre "Le

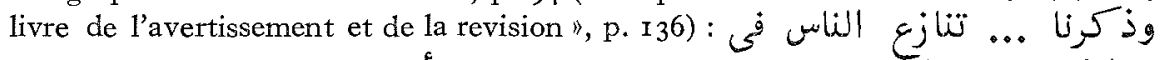

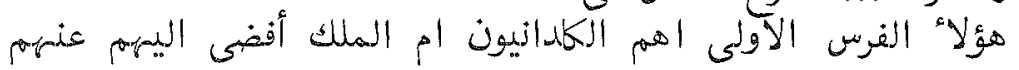

N.B. Une dispute $\left(\varepsilon^{j}{ }^{i}\right)$ a eu lieu sur cette question.

28 Voir ci-dessous, pp. 276 ss.

${ }_{29}$ Kitāb al-Muhabbar, p. 392 , ci-dessous, p. 285 n. 10.

${ }_{30}$ Cela semble s'ensuivre du fait que les yašt V, XV et XIX ne font pas mention de Gayōmart avant Hōšang dans la série d'adorateurs, malgré le fait que les premiers de ceux-ci y apparaissent dans le même ordre que dans l'histoire légendaire ultérieure où la première place pourtant revient à Gayōmart. Voir Gayōmart, chap. IV. Cf. Nyberg, Die Religionen des alten Iran, MVAG 43, 1938, pp. 391 et 398 contre p. 319; Wikander, Vayu I, pp. 6r s. contre p. 50.

31 Voir l'aperçu dans Christensen I, pp. I Io ss. La seule exception est peut-être la tradition que Mas'ūdī cite dans les Murūğ II, p. I I I (au sujet de Hōšang) : فهمنهم

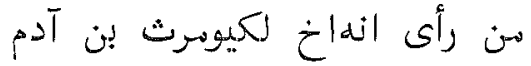

32 Bal'ami, Tarğamah-i Ta'rīh-i Tabarī; trad. de Zotenberg (Chronique de ... Tabari I), p. 5; Christensen I, p. 68. - Muṭahhar ibn Țāhir al-Maqdisī, Kìtāb al-bad'a wa al-ta'rīh, éd. Huart II, pp. I54 s., trad. II, pp. I4I s. (dans mon livre Gayōmart, pp. 134 et XVII). - Bīrūnī, Kitäb al-'ātār al-bāqiyah 'an al-qurün al-ḩāliyah, éd. Sachau sous le titre Chronologie orientalischer Völker, p. I03: on y donne un aperçu des rois persans. Gayōmart n'y figure pas expressément comme le père de Mašyag et Mašyānag, mais il est placé immédiatement avant eux. Au sujet de ces deux on dit : 
-Dārāb Pālan, Hulāsah-i Dīn, éd. Modi sous le titre The Persian Farziât-Nâmeh and Kholâseh-i Dîn, texte p. 49, trad. p. 66 : Mašyag et Mašyānag sont poussés du sperme de Gayōmart comme une seule forme

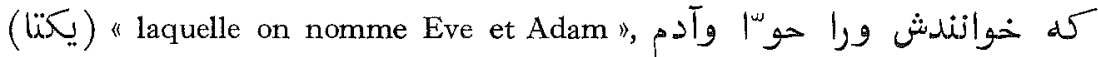

${ }_{33}$ Bìrünī, p. Io3; Muṭahhar ibn Ṭāhir al-Maqdisī II, pp. r54 s.

34 Ainsi Bal'ami.

${ }_{35}$ Voir ci-dessus la fin de la note 32.

${ }^{36}$ Rieu I, p. 69 a; Storey II, p. 6I.

37 Christensen I, p. 217.

s8 Christensen I, pp. 8I ss. et $2 \mathrm{I} 8$.

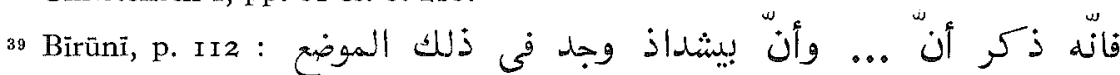

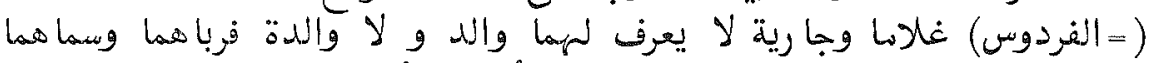

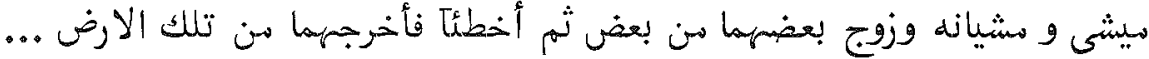

40 Cf. Christensen I, p. 89.

41 a) Auteurs ou savants persans:

Ṭabarī, Ta’rinh ..., éd. de Goeje sous le titre Annales quos scripsit ... at-Tabari, I,

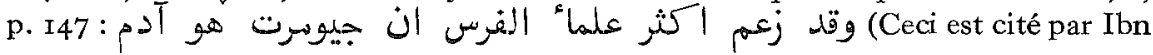
al-'Atīr, Ta'rīh al-kāmil, éd. le Caire I 303, p. I7.)

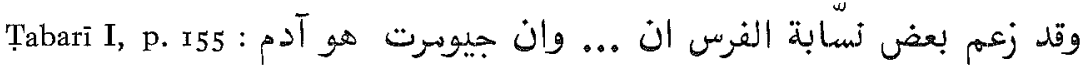

Bal'ami, trad. Zotenberg, p. 4 (Christensen I, p. 68) : "On rapporte aussi que le premier homme qui exista sur la terre fut Adam, qu'on désigne par le nom de Kayoumorth. C'est ce qu'attestent Mohammed-ben-Djehem, le Barmécide, Zadwiyyehben-Schahwiyyeh, le livre de Behram et celui des Sassanides, Mousâ-ben-Isâ, Khosrevi, Hâschem-ben-Qâsem Içfahânî, l'histoire des rois de Perse, et Ardavad Morghân, mobed des mobeds, qui a fait connaître l'histoire de Yezdeguerd. "(II nous semble assez évident qu'on doit examiner le manuscrit persan de ce texte de plus près.)

Ta'rîh-i Sistān, éd. Teheran I314, p. 2 : et p. 9 : (L'auteur anonyme croit lui-même que Gayömart soit identique à Adam.)

Muğmal al-tawã̄inh, éd. Teheran I3I8, p. 46r (au sujet de Gayōmart) : كتب فارسيان آدم شمرزلد

Dimišqī, Kitāb nuhbat al-dahr, éd. Mehren sous le titre Cosmographie de Chems-edDin Abou Abdallah Mohammed ed-Dimischqui, p. 256 (trad. par Mehren sous le titre Mamuel de la cosmographie du moyen âge, p. 370) : وذك بعض زمالبي الفرس

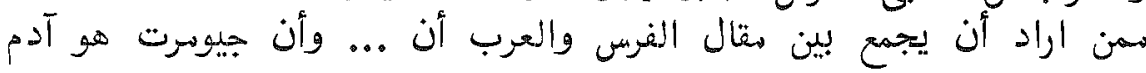

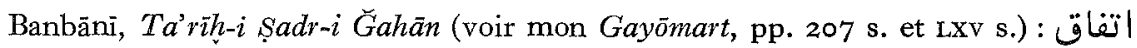

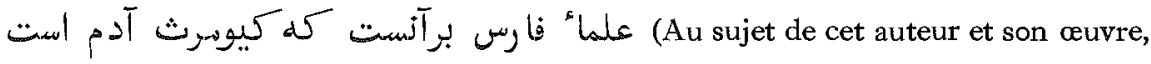
voir Rieu I, pp. 86 s., Blochet I, pp. 22I s., Storey II, pp. Io9 s.) 
b) Persans en général:

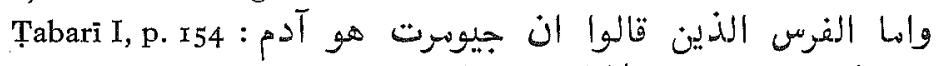

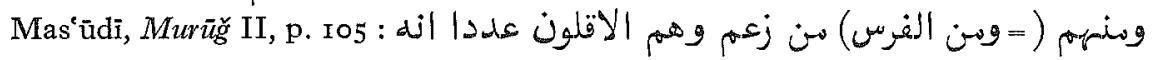

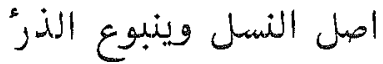

(Ici on ne dit pas directement que Gayōmart a été identifié àvec Adam, mais seulement qu'il a été regardé comme "l'origine de l'engendrement et la source de la propagation (du genre humain) ». Cette qualité pourrait revenir à Gayōmart même sans qu'il ait été identifié avec Adam. Ce que dit Mas'ūdī est valable pour toutes les identifications où Gayōmart garde la qualité de premier homme. Parmi celles-ci celle avec Adam est la plus importante. C'est pourquoi nous avons cité ici le passage en question. Christensen l'interprète seulement comme en rapport avec l'identification avec Adam. (Voir Christensen I, p. 87.) Ibn al-'Atir, I, p. I7 : بيونوت الذئى (Le contexte de ce passage indique pourtant qu'il s'agit de savants persans.)

Abū al-Fidā, $A l$-tawā̄inh al-qadīmat, éd. et trad. par Fleischer sous le titre $A b u l$ -

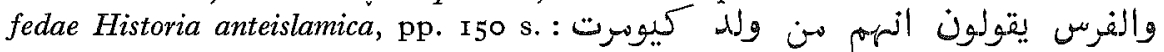

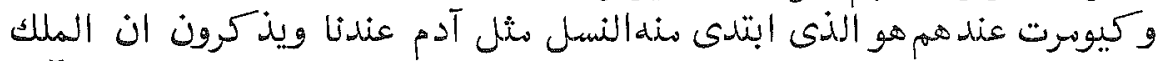
(Au sujet du début de cette citation, voir cidessus ce que nous avons dit à propos de Mas'ūdī, Murū̆ğ II, p. 105.)

Ibn Haldūn, Kitāb al-'ibar wa dīwān al-mubtada', éd. lith. II, pp. 5 s. (au sujet

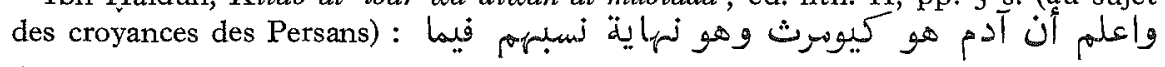
يزعمون

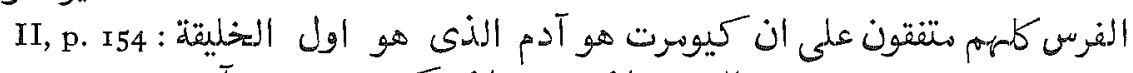

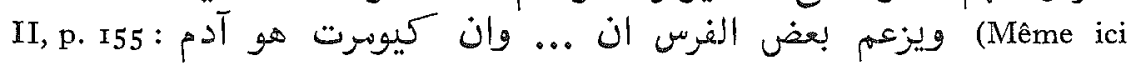
le contexte nous montre qu'il s'agit des vues des savants persans.)

Muntahab-i tawärīh-i $M u^{i} \bar{n} n \bar{\imath}$ (cité dans Gayōmart, pp. 194 et LVI) : J

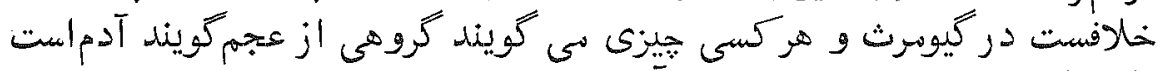

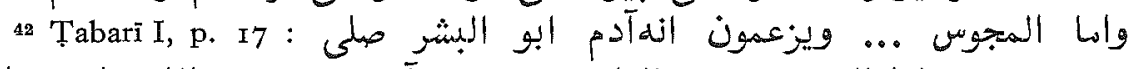

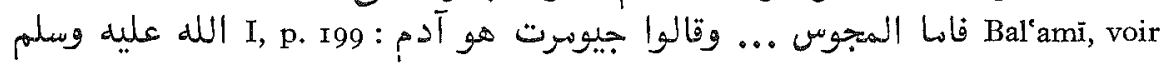
la note précédente.

Fãrsnāmah, éd. Le Strange et Nicholson sous le titre The Fársnámah of Ibnu

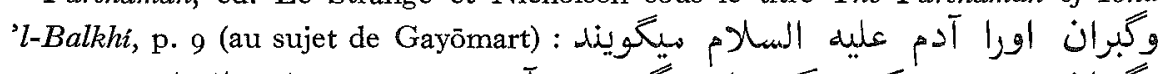

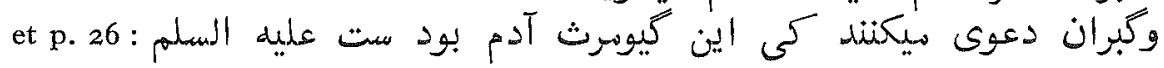

Šahrastāni, Kitāb al-milal wa al-nihal, imprimé dans la marge du livre Kitāb alfisal fĩ al-milal d'Ibn Hazm, le Caire I3I7-132I de l'Hégire, II, p. 73 :

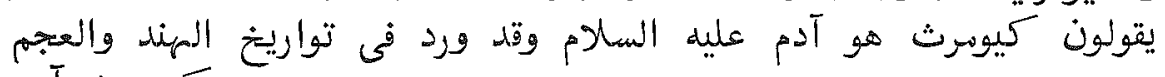
كيونرث آذئر

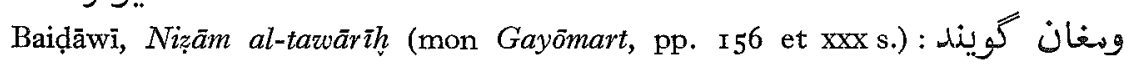
او آدماستق 
Nikpay ibn Mas'ūd, Histoire générale de la Perse et du monde musulman (le titre persan fait défaut dans le manuscrit), Fonds persan $6 \mathrm{I}$ ( = Blochet I, 253) fol. $7 \mathrm{v}^{\circ}$ :

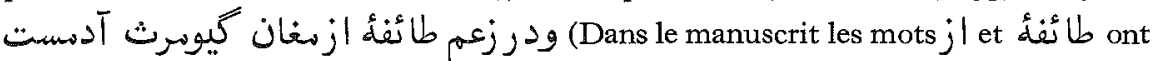
changé de place.) Sur l'auteur et son œuvre et sur notre manuscrit, voir Silvestre de Sacy dans Notices et extraits ... II, pp. 3 I5 ss.; Blochet I, pp. 200 s. et Storey II, p. 79 .

Banākatī, Ta'rīh hi Banākatī ou Raudat 'ūlì al-'albāb, selon mon livre Gayōmart,

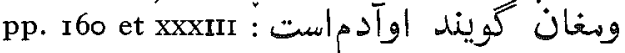

Faḍl Allāh Qazwĩnī, Kìtāb al-mu'ğam fī 'ātār mulūk al-'Ağam, selon mon livre

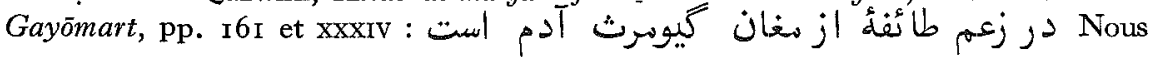
trouvons exactement les mêmes mots dans Häfiz-i Abrū, Mağma al-tazuārīh, Or. 2774 , fol. $29 \mathrm{r}^{\circ}$. (Ceci ne se trouve pas parmi ce que j'ai d'ailleurs cité de ce livre dans mon livre Gayōmart, pp. I 96 ss. et LVI ss.)

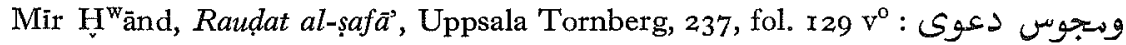
(Je n'ai pas eu à ma disposition les éditions de cette œuvre dont parle Storey II, pp. 95 ss.)

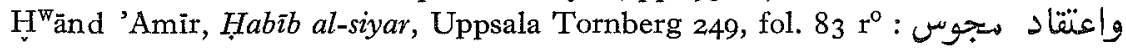

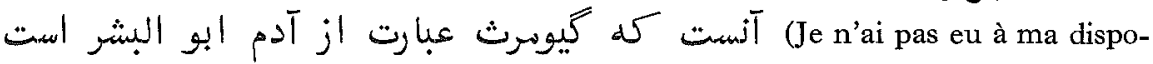
sition les éditions de cette œuvre dont parle Storey II, pp. Io6 ss.)

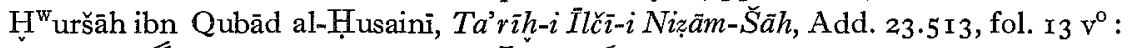
(Sur l'auteur et son œuvre, voir Storey II, pp. I I3 s., et sur notre manuscrit, voir Rieu I, pp. IO7 ss.)

${ }^{43} \underline{T a}^{*} a \bar{l} l i b i$, Gurar 'ahbār mulūk al-Furs, éd. et trad. par Zotenberg sous le titre Histoire des rois des Perses, p. I : اختلف الروّاة سن اصناف الاسم فيل اختلاف

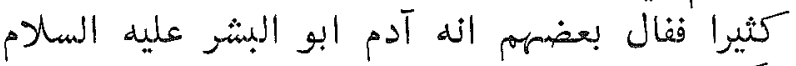

اصيحاب تواريخ اختانف كردمانل : (Sur l'auteur et son œuvre, voir Storey II, p. 89, et sur notre manuscrit, voir Rieu, Suppl., pp. 270 s.)

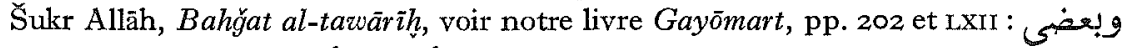

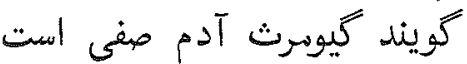

${ }_{44}$ Dârâb Hormazyâr's Rivâyat (éd. Unvâlâ) I, pp. 256 s. Dans la version parsie de

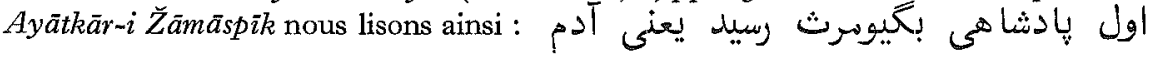
(Le texte se trouve dans Modi, Pahlavi Translations III, p. 86. Il y a d'autres variantes, voir Dhabhar, The Persian Rivayats, p. 489 n. 5 et Dârâb Hormazyâr's Rivâyat II, p. I05.)

${ }^{45}$ Pp. 2 et 9. Les passages sont cités ci-dessus, n. 4I.

${ }_{46}$ N. 4 I.

${ }_{47}$ Muhammedanische Studien I, pp. IOI-216, spécialement pp. I77-207. Cf. aussi Browne, A Literary History of Persia I, pp. 265 ss.

48 Mon Gayōmart, pp. ${ }_{35}$ ss. et XVIII ss.

49 Gayōmart, pp. I36 et 140 ; pp. XVIII et XXI. 


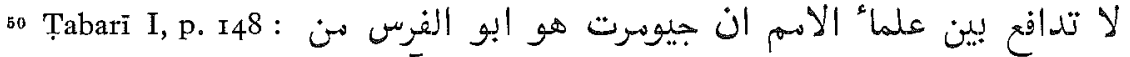

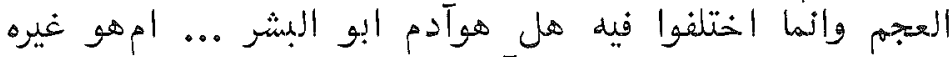

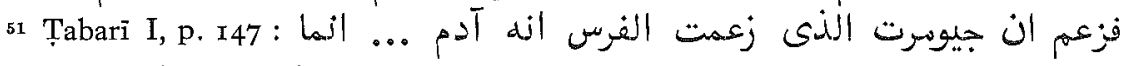

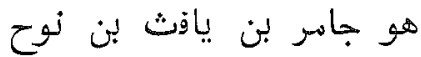

52 Voir ci-dessus, n. 4I.

${ }^{53}$ Voir ci-dessus, n. 4I.

54 Voir ci-dessus, n. 4I.

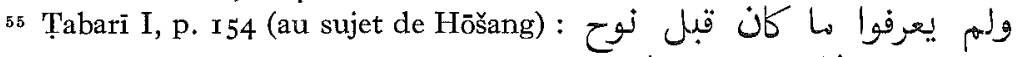

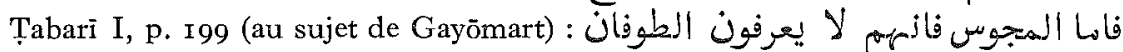

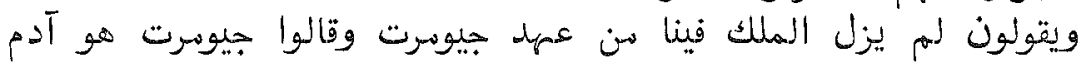

56 Ṭabarī I, pp. I54 s.

57 Ṭabarī I, p. 154 :

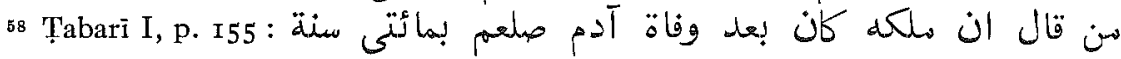

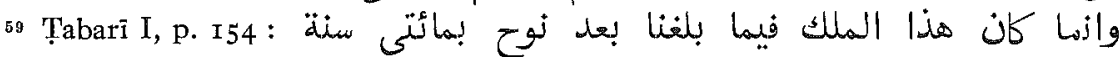
فُصيره اهل فالى فارس بعدآدم بمائتى سنة

${ }_{60}$ Christensen I, pp. I24-130.

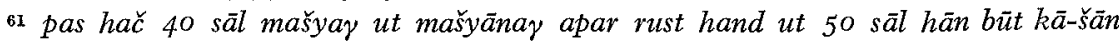

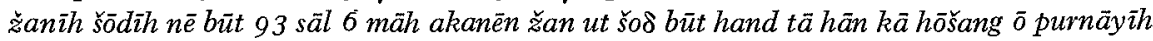
mat, "Après 40 ans Mašyag et Mašyānag poussèrent. 5o ans furent (la durée du temps) où leur qualité de femme et homme n'existait pas. Pendant 93 ans et 6 mois ils existaient ensemble comme femme et homme jusqu'à ce que Hōšang atteignit l'âge de majorité. " (Nous avons ici combiné le texte de Bundahišn $\mathrm{A}$ avec celui de Bundahišn J.)

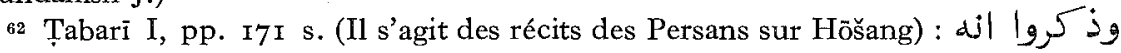
... وانه كان بين موت جيومرت الى مولد اوشهنج وملكه مائتا سنة وثلث وعشرون سنة

وقد ذهب:

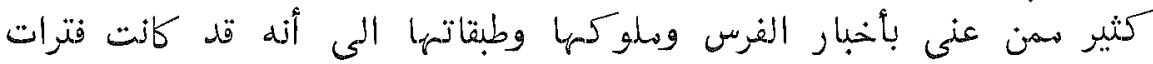

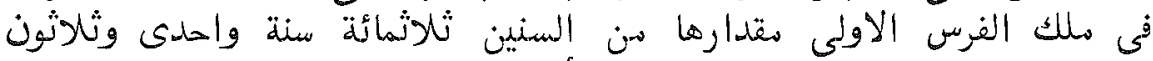

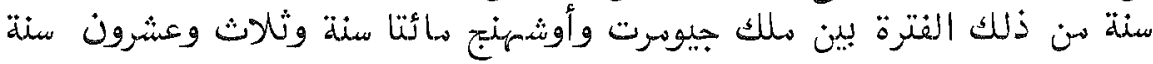

${ }_{64}$ Bibliothèque Orientale III, p. 248.

${ }_{65}$ Christensen II, pp. 8I s.

55 Ibidem.

67 Voir ci-dessus, p. 270 et n. 4 I.

68 Goldziher, Muhammedanische Studien I, pp. I46, I66 et $174 \mathrm{~s}$.

${ }^{69}$ Voir ci-dessus, p. 268 et n. 4I. Le Ta'rīh $-i$ Siststän contient des matériaux de plusieurs sources anciennes, parmi lesquelles on note le Bundahišn qui s'appelle كن.

${ }_{70}$ Voir ci-dessus, n. 42.

${ }^{11}$ Voir ci-dessus, n. $4 \mathrm{I}$.

${ }^{2}$ Voir ci-dessus, n. 41. 
73 Voir ci-dessus, n. 4r.

74 Voir ci-dessus, n. 4I.

75 C.-à-d. du quatorzième siècle environ. Aucun auteur persan de cette époque, que je sache, n'accepte personnellement l'identification de Gayōmart avec Adam, et personne ne dit que les Persans en général ne l'ont fait. Pour la forme je fais ici mention des auteurs persans du quatorzième siècle dont $\mathrm{j}$ 'ai examiné la position dans ce sujet : Nikpay ibn Mas'ūd (ci-dessus, n. 42), Banākatì (ci-dessus, n. 42), alHarawi, Ḥamd Allāh Mustawfī, Faḍl Allāh Qazwīni (ci-dessus, n. 42) et Šabānkāra’ī. D'ailleurs je voudrais souligner le fait qu'aucun des auteurs islamiques persans après l'année $95^{\circ}$ (sauf l'auteur du Ta'rīh-i Sĩstân) cités dans cet article, ne confirme ce que disent les trois auteurs arabes mentionnés ci-dessus.

76 Voir ci-dessus, pp. 27 I s.

77 Voir ci-dessus, p. 27 I et n. 55 .

${ }_{78}$ TTabari I, p. 199, cité déjà ci-dessus n. 55.

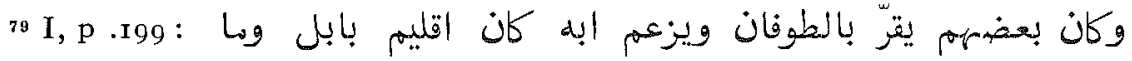

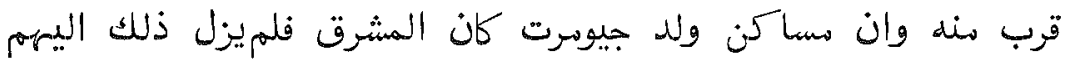

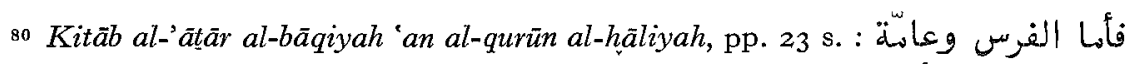

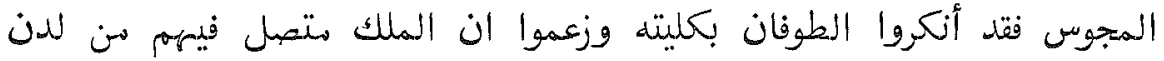

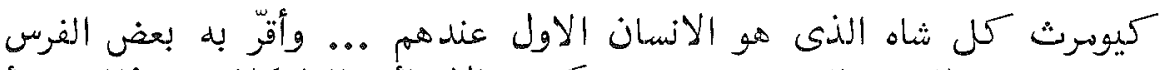

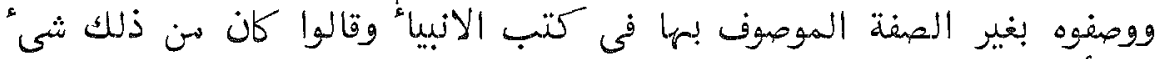

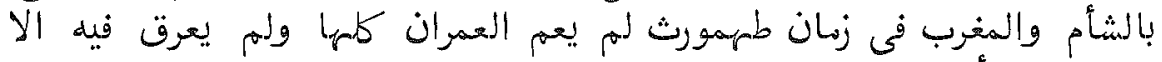

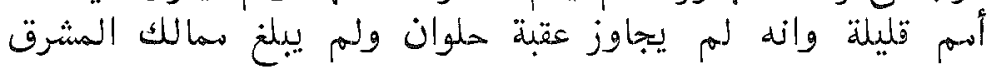

فوجل فيل : Hamzah, éd. Calcutta, p. I74; éd. Gottwaldt p. I99, trad. p. I53

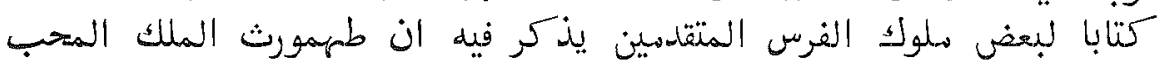

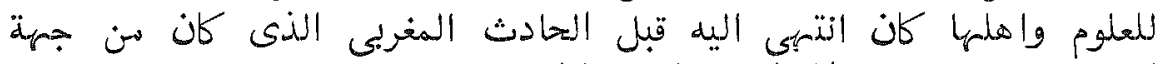

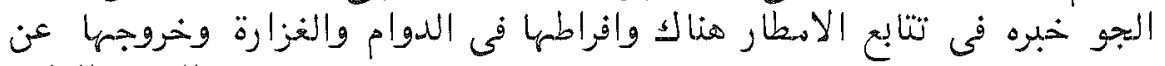

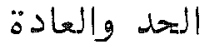

82 Voir ci-dessus, p. 266 et n. 22 et n. 23.

83 Il y a une tradition chrétienne disant que le déluge ne s'étendait pas sur le paradis. Voir la citation d'Afrem chez Georgius Syncellus, p. 26, et en outre les récits des deux livres "Die Schatzhöhle " et "The Book of the Bee".

84 I, pp. I $99 \mathrm{~s}$.

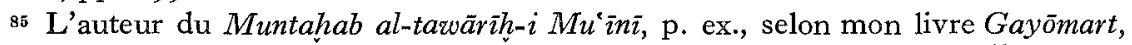

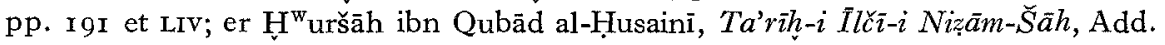
23.5 I3, fol. I4 $\mathrm{r}^{\circ}$ (la marge).

وجعلنا ذريته هم الباقين

فاخبر عز زكره ان ذزية نوح هم الباقون دون غيرهم

88 Voir n. 83 .

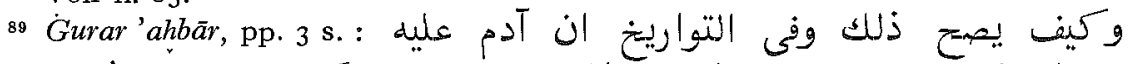

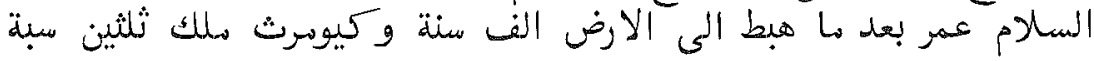




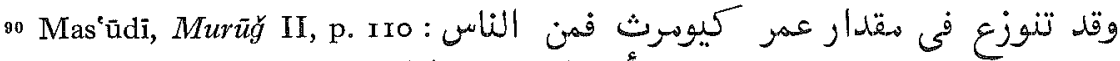

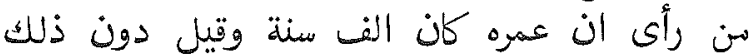

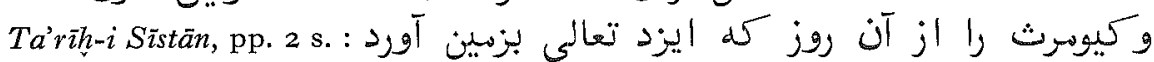

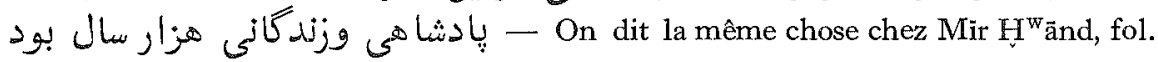
I3 I r r $^{\circ}$, et $\mathrm{H}^{\mathrm{w}}$ ānd 'Amir, fol. $83 \mathrm{r}^{\mathrm{o}}$. Cf. aussi d'Herbelot II, p. I79.

91 Christensen $\mathrm{I}$, pp. $\mathrm{r} 28 \mathrm{~s}$.

92 Ibn Qutaiba, Kitāb al-måārif, éd. le Caire en ז300, p. I9: وعاش آدم صلى Tabari (I, p. I55) calcule la durée de la vie de Mahalaleel selon " ce que l'on a raconté de l'Apôtre d'Allāh ... en ce qui concerne la vie d'Adam, (à

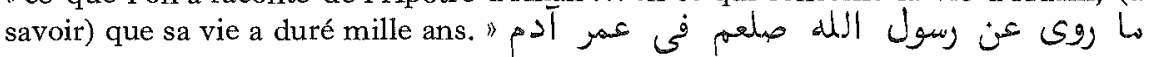
(il - Ces mille ans semblent d'origine juive. Voir Sidersky, Les origines des légendes musulmanes, pp. I9 s., où l'on traite la légende selon laquelle Adam a donné à David 40 (ou 70 ) années de sa vie de mille ans. Des versions de cette légende se trouve chez Tabarī I, pp. I 55 ss. On dit pourtant qu'Allāh a

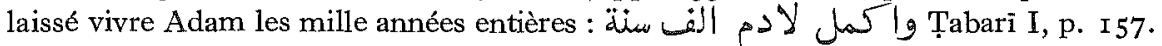

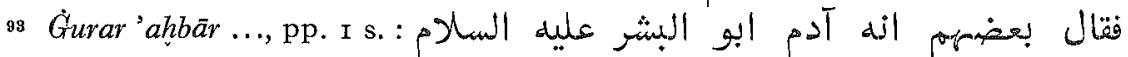

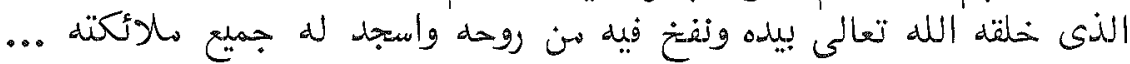

Cf. le Coran XXXVIII, 7 I ss. et VII, Io ss. — Sur l'origine juive de cette conception islamique, voir Sidersky, pp. 9 ss.

94 Voir le texte persan dans mon livre Gayōmart, pp. 202 et LXI : بعضى عجم

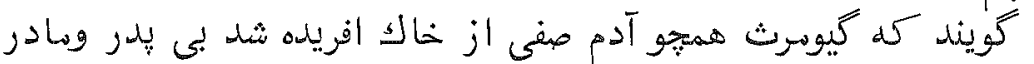

Cf. le Coran XXXVIII, 7I, et VII, Ir. - Cette conception pourrait pourtant être d'origine iranienne, car le Bundahišn A dit (p. 2I) : "Et il (c.-à-d. Ōhrmazd) créa de la terre Gayōmart avec le Bœuf. " (ut-aš gayōmart apāk gäv hac zamīk âfrīt) Dans notre livre Gayōmart nous avons (pp. II 5 et IV) cité un autre texte pehlevi (PRDD, pp. I36 s.) où l'on dit que les hommes sont créés de l'argile (gil) d'où vient Gayōmart. - Même Sukr Allāh dit que Gayōmart a poussé de la terre (voir Gayō-

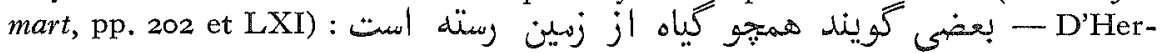
belot raconte d'après Caiumarath Nameh qu'Adam séparé une fois longtemps d'Eve eut une pollution nocturne, "de sorte que la semence féconde de ce premier pere des hommes étant tombée à terre, il s'en forma une plante qui prit la figure humaine, $\&$ devint enfin le Caiumarath dont nous parlons. "(Bibl. Or. II, pp. I8I s.)

95 Tabarī I, p. I47; Ibn al-'Atīr, p. I7.

Mutahhar ibn Țāhir al-Maqdisĩ II, pp. I54 s., trad., pp. $x_{4}$ I s.

Ğūž̆ānī, selon mon Gayōmart, pp. 155 et xxx.

Šabānkāra'ì, selon mon Gayōmart, pp. I 86 et L et LI.

Harawi, Ta'rīh-nāmah-i Harāt, p. 5 I.

Muntahab-i tawā̄īh-i Mu'iñi, selon mon Gayōmart, pp. rgI et LIV.

Mūsawi, Ta'rìh-i hairāt, Or. 4898 , fol. $8 \mathrm{v}^{\circ}$.

Kühistāni, selon mon Gayōmart, pp. 208 et LXVI.

Ahmad ibn Bahbal, selon mon Gayōmart, pp. 2II et LXIX.

96 'Ta'ālibì, p. 2. - Ǵazzālì, Nașinhat al-mulūk, éd. Ğalāl Humā’ì, Téhéran I 3I7, p. 42. On cite souvent Gazzālī comme garant de cette conception. Ainsi Baiḍāwĩ, 
Banākatī, Fạ̣l Allāh, Ḥāfiz̨-i Abrū. Voir les textes des dits auteurs dans mon livre Gayōmart.

97 Mas'ūdī, Muruğ II, p. I05. Mīr $\mathrm{H}^{\mathrm{w}} \overline{\mathrm{a}} \mathrm{n}$, fol. I29 $\mathrm{r}^{\circ}$.

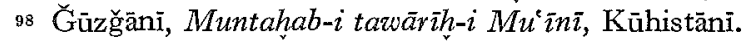

${ }_{99}$ Muntahab-i tawärīh-i Müiñ

100 Voir n. 96.

101 Mir $\mathrm{H}_{v}^{\mathrm{w}} \bar{a}$ d, fol. $129 \mathrm{r}^{\mathrm{O}}$. Nous ne pouvons pas voir que Gazzāli dit ceci expressément dans le livre Nașịhat al-mulük.

102 Nous avons traité cette question dans notre livre Gayōmart, p. I 86 n. 4.

${ }_{103}$ Voir n. $9^{8}$ que l'on peut compléter avec Šabānkāra'î, quoique les enfants de Caïn y soient des enfants apostats d'Adam.

104 Les deux versions de Muntahab-i tawärīh-i $M u^{e} i ̄ n \overline{\text {, }}$, voir Gayömart, pp. 191-196, LIV-LVI.

105 Voir mon article "Die vorflutlichen Chaldäer in arabischer Überlieferung ", Orientalia Suecana XIV-XV, I965-1966, p. 95 (selon Bīrūnī).

106 Voir ci-dessus, pp. 265 ss.

107 Voir $A l-Q \bar{a} n \bar{u} n$ al-mas' $\bar{u} d \bar{\imath}$. Le passage en question est traduit dans notre article

dans Orientalia Suecana que nous venons de mentionner, p. 95. Chez Ğüzğānī nous avons la date erronée I024 au lieu de ro64. Voir mon Gayömart, p. I55 n. I.

108 Voir l'article dans Orientalia Suecana et la littérature que nous y indiquons. (Spécialement les livres de Gelzer et Schnabel.) Voir en outre Eusèbe, coll. I9, 23.

${ }_{109}$ Ta älibì.

ıo Muțahhar ibn Țāhir al-Maqdisi, Ğūzğāni.

111 Tabarī, Ibn al-'Atīir, Mas'ūdī, $H_{\vee}{ }^{\mathrm{W}}$ ānd 'Amīr.

112 Ğūẓ̆ānì.

113 P. 42.

114 Voir Gayömart, pp. I42 et XXII.

115 Voir n. 95.

116 Voir n. 95.

117 Geschichte von Tabaristan, Rujan und Masanderan, éd. Dorn, Saint-Pétersbourg I 850 , pp. I 47 et I 54 .

118 Voir n. 95.

119 Voir n. 95.

120 Gayömart, pp. 142 et xxII.

121 Muğmal at-tawārīh, p. 23 (قونى

122 يونانيان, Šukr Allāh, selon mon Gayōmart, pp. 202 et Lxir.

123 Gayōmart, pp. 202 et LXI.

124 Voir Ğüzğānī, p. ex., selon Gayōmart, pp. I53 s. et XxIx.

125 Gayōmart, pp. 202 et Lxrr.

${ }_{126} \mathrm{Bal}^{\mathrm{i}}$ ami selon Lazard dans Fournal Asiatique 1956, pp. 201-216. Des rapports insignificants en ce qui concerne l'origine de cette identification se trouvent chez

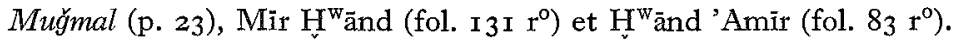

127 Lazard dans Fournal Asiatique 1956, pp. 201-216.

${ }_{128}$ Bibliothèque Orientale II, p. I79.

${ }_{129} \mathrm{Bal}^{\prime}$ ami, trad. par Zotenberg I, p. Ioo. Šukr Allāh, selon Gayōmart, pp. 202 et LXII.

${ }^{130}$ Faḍl Allāh, selon Gayōmart, pp. I6I et xxxıv, Ḥăfiz-i 'Abrū, Or. 2774, fol. $29 \mathrm{r}^{\circ}$. 
131 Bānbānī, selon Gayōmart, pp. 207 et Lxv, Cf. aussi d'Herbelot, Bibl. Or. V, p. I07.

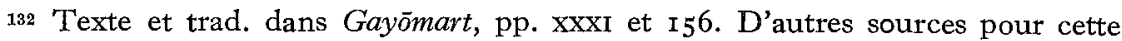
identification : Fạ̣l Allāh (Gayōmart, pp. I6r et xxxıv), Sabānkāra’ĩ (Gayõmart, pp. I86 et L), Bānbānī (Gayōmart, pp. 207 et LXV).

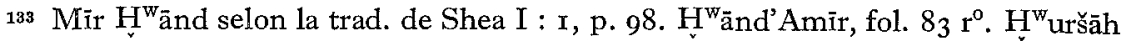
ibn Qubäd al-Husainī, fol. $13 \mathrm{v}^{\circ}$. D'Herbelot II, p. I79.

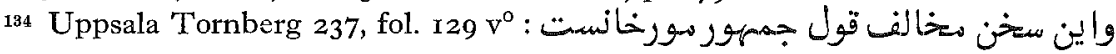

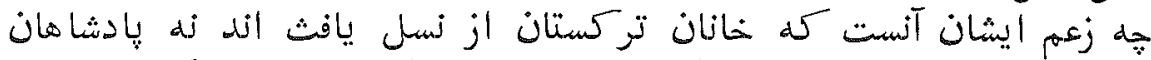

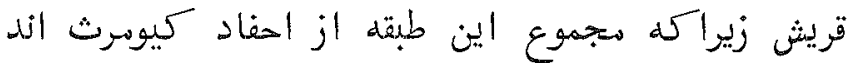

135 Ceci a naturellement des rapports avec la conception de Gayōmart en tant que premier roi. (Cf. ci-dessus, p. 276 p. ex.) Ǵazzāli (et même d'autres) nomme Gayōmart le premier roi et dit que son pouvoir concerne ce monde-ci, tandis que celui de Seth concerne l'autre monde. Nașinhat al-mulük, p. 42.

136 Voir ci-dessus, pp. $269 \mathrm{~s}$.

137 I, p. 2 IO.

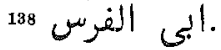

139 Voir p. 275 et n. 90-92.

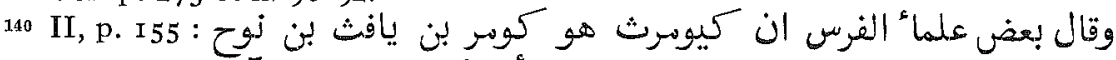

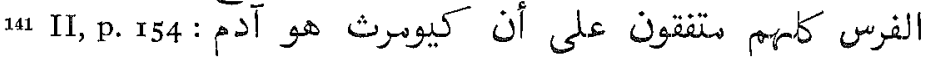

142 Christensen I, p. 87.

143 Gayōmart, pp. I40 s.

144 Murüğ II, p. I05 : طائفة

145 Ğūzğāni, selon Gayömart, pp. I55 et xxx.

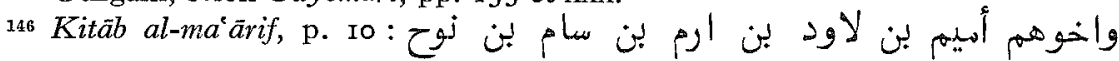

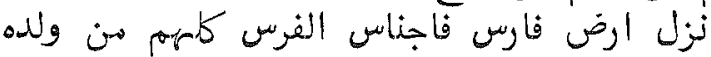

${ }_{147}$ Cf. Mas'ūdì, Murū̌̆ I, p. 78; II, p. I05.

${ }_{148}$ Voir Kitāb al-ma'ärif, p. 220, p. ex. Voir en outre Nöldeke, Geschichte der Perser und Araber zur Zeit der Sassaniden, p. XxI n. 2, Christensen I, p. 65.

${ }_{149}$ Browne, $A$ Literary History of Persia I, p. 268. Goldziher, Muhammedanische Studien I, pp. 147-216.

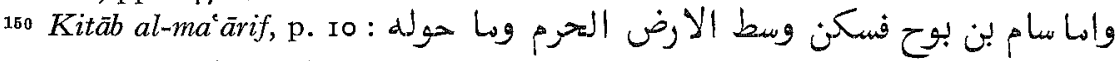

Cf. Maseũiĩ, Murūğ I, p. 77.

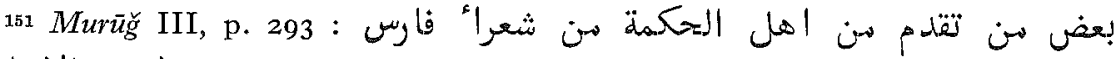

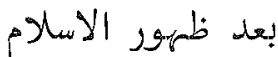

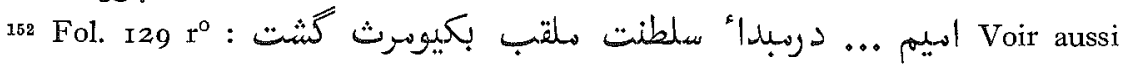
$H^{\mathrm{w}}$ ānd'Amir, fol. $83 \mathrm{r}^{0}$.

${ }_{153}$ Muruğ III, p. 292. D’autres sources : Muğmal, p. 23; Muntahab-i tawārīh-i $M u^{`} \bar{\imath} n \bar{i}$, selon Gayōmart, pp. I92 et LIV.

Je tiens à remercier M. Emile Morin, Anzême, d'avoir bien voulu corriger le français de cet article. 